\title{
SIMULTANEOUS BIFURCATION OF LIMIT CYCLES FROM A CUBIC PIECEWISE CENTER WITH TWO PERIOD ANNULI
}

\author{
LEONARDO P. C. DA CRUZ AND JOAN TORREGROSA
}

\begin{abstract}
We study the number of periodic orbits that bifurcate from a cubic polynomial vector field having two period annuli via piecewise perturbations. The cubic planar system $\left(x^{\prime}, y^{\prime}\right)=\left(-y\left((x-1)^{2}+y^{2}\right), x\left((x-1)^{2}+y^{2}\right)\right)$ has simultaneously a center at the origin and at infinity. We study, up to first order averaging analysis, the bifurcation of periodic orbits from the two period annuli, first separately and second simultaneously. This problem is a generalization of [24] to the piecewise systems class. When the polynomial perturbation has degree $n$, we prove that the inner and outer Abelian integrals are rational functions and we provide an upper bound for the number of zeros. When the perturbation is cubic, the same degree as the unperturbed vector field, the maximum number of limit cycles, up to first order perturbation, from the inner and outer annuli is 9 and 8 , respectively. When the simultaneous bifurcation problem is considered, 12 limit cycles exist. These limit cycles appear in three types of configurations: $(9,3),(6,6)$ and $(4,8)$. In the non-piecewise scenario, only 5 limit cycles were found.
\end{abstract}

\section{INTRODUCTION}

The knowledge of the existence of periodic solutions is very important for understanding the dynamics of differential systems. The method of averaging has a long history that starts with the classical works of Lagrange and Laplace who provided an intuitive justification of the mechanism. The first formalization of this procedure was given by Fatou in 1928, see [8]. Nevertheless, Buica and Llibre [1] extended the averaging theory for studying periodic orbits to continuous differential systems using mainly the Brouwer degree theory. Recently, the averaging theory for studying periodic orbits to piecewise differential systems has been developed, see $[16,17]$ for example. Here we use the same approach as [2].

Consider the perturbed polynomial piecewise differential system

$$
Z^{ \pm}=\left\{\begin{array}{l}
\dot{x}=-y\left((x-1)^{2}+y^{2}\right)+\varepsilon P_{n}^{ \pm}(x, y), \\
\dot{y}=x\left((x-1)^{2}+y^{2}\right)+\varepsilon Q_{n}^{ \pm}(x, y),
\end{array} \quad \text { if }(x, y) \in \Sigma^{ \pm},\right.
$$

with $P_{n}^{ \pm}$and $Q_{n}^{ \pm}$polynomials of degree $n$ and $\Sigma^{ \pm}=\{(x, y): \pm y>0\}$. An example of the phase portrait of the above system, for $\varepsilon$ small, is drawn in Figure 1.

Following [2], the limit cycles of (1) correspond to the zeros of the difference map $\Pi^{+}(r)-\left(\Pi^{-}\right)^{-1}(r)$, see Figure 2. Moreover, for $\varepsilon$ small enough and doing a time rescaling, the simple zeros of $I(r)=I^{+}(r)-I^{-}(r)$, where

$$
I^{ \pm}(r)=\int_{\gamma_{r}^{ \pm}} \frac{P_{n}^{ \pm}(x, y) d y-Q_{n}^{ \pm}(x, y) d x}{(x-1)^{2}+y^{2}},
$$

gives limit cycles for (1), bifurcating from $\gamma_{r}^{ \pm}=\left\{x^{2}+y^{2}=r^{2}: \pm y>0\right\}$. The above integrals defined over closed curves are known as Abelian integrals, see [4]. We can say that the expression (2) are the piecewise version of them. See more details in [11] or

2010 Mathematics Subject Classification. Primary 34C07, 34C23, 37C27.

Key words and phrases. Piecewise vector field, Limit cycles, Simultaneous bifurcation, Zeros of Abelian integrals. 


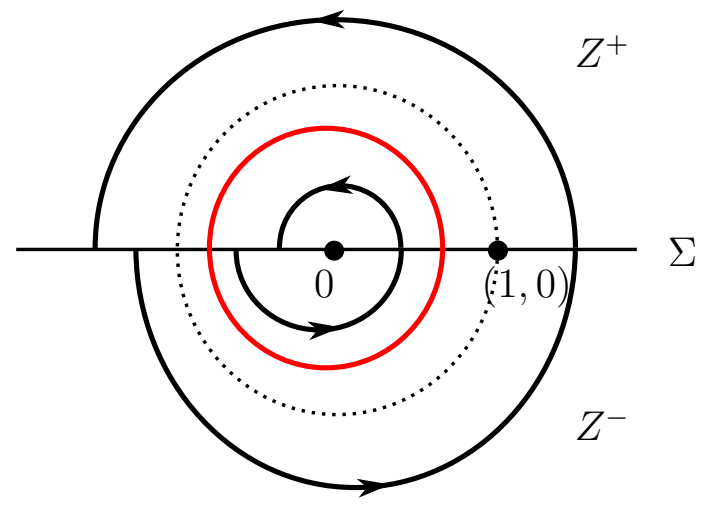

Figure 1. A possible phase portrait of system (1)

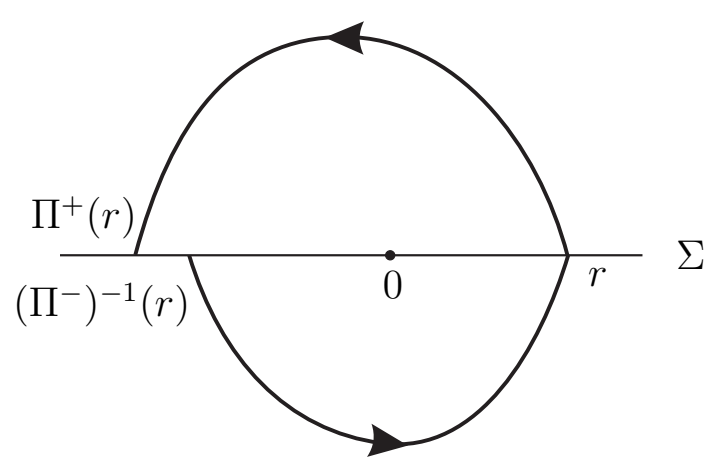

FIGURE 2. Return map for system (1)

[19]. In our case both components of the unperturbed system have a common factor that appears in the denominator of the integrand. This expression appears in [18] (in polar coordinates) or in [10]. As we will see in Theorem 1.2 the explicit expression of (2) is different in the two period annuli associated to (1):

$$
\mathcal{R}_{i}=\{r \in \mathbb{R}: 0<r<1\} \text { and } \mathcal{R}_{e}=\{r \in \mathbb{R}: r>1\} .
$$

As we have commented before, the function $I(r)$ is also called the Abelian integral associated to system (1). By similarity we define the inner and outer Abelian integrals as

$$
\begin{aligned}
& I_{i}(r)=I_{i}^{+}(r)-I_{i}^{-}(r), \quad 0<r<1, \\
& I_{e}(r)=I_{e}^{+}(r)-I_{e}^{-}(r), \quad r>1,
\end{aligned}
$$

where $I_{j}^{ \pm}$are the upper and lower inner $(j=i)$ and outer $(j=e)$ integrals.

The study of the number limit cycles that bifurcate from a linear center, also called harmonic oscillator, is very relevant in the qualitative theory of differential equations. Over the last two decades, there have been papers showing that when we add a curve of singularities the number of limit cycles appearing by perturbation increases. In [18], only doing a first-order analysis, it was proved that this number is doubled, adding a straight line of singularities to the unperturbed system, in comparison with the perturbation of the linear center. Similar results have been done adding curves with a fixed degree. See, for example, [10] for the study of a fixed number of straight lines or [25] for a circle of singularities. But, there are not so many papers focused on the study of simultaneous bifurcation of limit cycles from centers with different period annuli. Some of them are $[6,9]$ that deal with the simultaneity in two different regions, or [7] where three separated period annuli appear. A study of the bifurcation of limit cycles from different period 
annuli of polynomial Hamiltonian systems to obtain lower bounds for the Hilbert number is done in [5] and, more recently, in [14]. Although the most common technique to study simultaneity is the $Z_{n}$ symmetry, see for example [20,21], when it is not considered, see for example [3], more periodic orbits appear. This is the case done in [24] where nonsymmetric perturbations are considered. Following the same procedure we consider now a piecewise polynomial perturbation of the two nested period annuli. The main goals are consequence of the explicit expressions for the Abelian integrals that we have obtained. From this fact, we can study the upper bound of the number of zeros in both regions separately and the existence of some configurations of simultaneity. We will show also that, in some sense, there are no others.

The explicit expression obtained for the functions (2) allow us to use the classical theory of Chebyshev systems to provide an upper bound for the number of zeros. Now we recall the main definitions and properties. Let $\mathcal{F}=\left[f_{0}, \ldots, f_{n}\right]$ be an ordered set of functions of class $\mathcal{C}^{n}$ defined in a closed interval $[a, b]$. We consider only elements in $\operatorname{Span}(\mathcal{F})$, that is, functions such as $f=a_{0} f_{0}+a_{1} f_{1}+\cdots+a_{n} f_{n}$ where $a_{j}$ for $j=0,1, \ldots, n$ are real numbers. We say that $\mathcal{F}$ is an Extended Chebyshev system, ET-system in short, on $[a, b]$ if the maximum number of zeros, taking into account its multiplicity, is $n$. For a sufficient condition we can introduce the Extended Complete Chebyshev systems. We say that $\mathcal{F}$ is an Extended Chebyshev system, ECT-system in short, on $[a, b]$, if any set $\left[f_{0}, \ldots, f_{j}\right]$, for $j=0, \ldots, n$ is an ET-system on $[a, b]$. When all the Wronskians, $W_{j}=W\left(f_{0}, \ldots, f_{j}\right)$ $j=0, \ldots, n$, are different from zero in $[a, b]$ the family $\mathcal{F}$ is an ECT-system. More details on ET-systems and ECT-system can be found in [12]. Here we use an extension of this theory, the extended Chebyshev systems with accuracy (see [23]), because some Wronskians vanish.

Before presenting our results we state a definition about what we consider that a configuration of limit cycles is.

Definition 1.1. We say that the system (1) presents a configuration with exactly $(k, \ell)$ limit cycles when it has $k$ and $\ell$ limit cycles in $\mathcal{R}_{i}$ and $\mathcal{R}_{e}$, respectively.

Next result provides the general expressions for the first averaged functions in the inner and outer period annuli. Moreover, writing these functions using an Extended Complete Chebyshev system we can also find an upper bound for the number of zeros and, consequently, for the number of limit cycles, up to a first order analysis in $\varepsilon$, that system (1) has.

Theorem 1.2. Let $P_{n}^{ \pm}$and $Q_{n}^{ \pm}$be polynomials of degree $n$ in (1). Then, the inner and outer Abelian integrals (3) associated to (1) are

$$
\left\{\begin{array}{l}
I_{i}(r)=\frac{r R_{2 n+1}(r)}{r^{2}-1}+H_{n}\left(r^{2}\right) \log \left(\frac{1-r}{1+r}\right) \text { if } 0<r<1, \\
I_{e}(r)=\frac{r S_{2 n+1}(r)}{r^{2}-1}+H_{n}\left(r^{2}\right) \log \left(\frac{r-1}{1+r}\right) \text { if } r>1,
\end{array}\right.
$$

where $R_{m}, S_{m}$ and $H_{m}$ are polynomials of degree $m$. Moreover, the maximum number of zeros of each $I_{i}$ and $I_{e}$ is $4 n+8$.

For fixed (small) values of $n$, the above upper bound is far to be optimal. A more precise study can be done for the cubic family when both period annuli are considered separately and also when the simultaneity is taken into account. Next theorem gives our main result for cubic perturbations.

Theorem 1.3. For $n=3$, the functions $I_{i}(r)$ and $I_{e}(r)$, defined in (4), have at most 9 and 8 zeros, respectively. Moreover, there exist polynomial perturbations such that (1) 
exhibits configurations with 12 limit cycles. In particular it presents configurations with $(9,3),(6,6)$ and $(4,8)$ limit cycles.

The perturbation inside the piecewise polynomial class of degree $n$, including the proof of Theorem 1.2, is presented in Section 2. The rest of the paper is devoted to the cubic family. In Section 3 we study each region, $\mathcal{R}_{i}$ and $\mathcal{R}_{e}$ separately. The first statement of Theorem 1.3 is also proved here. The simultaneity study and the rest of the proof of Theorem 1.3 is done in Section 4. Additionally, also in Section 4, we study some bifurcation diagrams where the different configurations can appear. Moreover, we also provide other configurations with less number of limit cycles. In particular, as we are working in a 10-dimensional space of parameters, the technique used in Section 4 provides all the configurations $(k, \ell)$ with $k+\ell=10,0 \leq k \leq 8$ and $0 \leq \ell \leq 7$. Finally, in Section 5, we study the local behavior of the bifurcation curves near the boundary of the domain of definition.

\section{The GENERAL CASE}

This section is devoted to the proof of Theorem 1.2. The first statement follows from the explicit computation of the Abelian integrals defined in the two annular regions $\mathcal{R}_{i}$ and $\mathcal{R}_{e}$. The second statement is proved using ECT-systems, see [12].

As in [24], the integrals (2), in the usual polar coordinates $(x, y)=(r \cos \theta, r \sin \theta)$, can be written, for $j \in\{i, e\}$, as

$$
\begin{aligned}
I_{j}^{ \pm}(r) & =\sum_{m=1}^{n+1} r^{m} \int_{0}^{ \pm \pi} \frac{\sum_{k=0}^{m}\left(\alpha_{k, m}^{ \pm} \cos (k \theta)+\beta_{k, m}^{ \pm} \sin (k \theta)\right)}{r^{2}-2 r \cos \theta+1} d \theta \\
& =\sum_{k=0}^{n+1} r^{k} R_{k}^{ \pm}\left(r^{2}\right) C_{j, k}^{ \pm}(r)+\sum_{k=1}^{n+1} r^{k} T_{k}^{ \pm}\left(r^{2}\right) S_{j, k}^{ \pm}(r),
\end{aligned}
$$

where

$$
\begin{aligned}
& C_{i, k}^{ \pm}(r)=\int_{0}^{ \pm \pi} \frac{\cos (k \theta) d \theta}{r^{2}-2 r \cos \theta+1}, 0<r<1, \\
& S_{i, k}^{ \pm}(r)=\int_{0}^{ \pm \pi} \frac{\sin (k \theta) d \theta}{r^{2}-2 r \cos \theta+1}, 0<r<1, \\
& C_{e, k}^{ \pm}(r)=\int_{0}^{ \pm \pi} \frac{\cos (k \theta) d \theta}{r^{2}-2 r \cos \theta+1}, r>1, \\
& S_{e, k}^{ \pm}(r)=\int_{0}^{ \pm \pi} \frac{\sin (k \theta) d \theta}{r^{2}-2 r \cos \theta+1}, r>1 .
\end{aligned}
$$

Moreover, $\alpha_{k, m}^{ \pm}=\beta_{k, m}^{ \pm}=0$ when $k$ and $m$ do not have the same parity and $R_{k}^{ \pm}\left(r^{2}\right)$ and $T_{k}^{ \pm}\left(r^{2}\right)$ are polynomials of degree at most $[(n+1-k) / 2]$ with arbitrary coefficients for all $k=0, \ldots, n+1$.

We start proving Lemma 2.1, that provides the recurrence to compute the integrals given in (5). In Lemma 2.2 we provide the explicit expressions for (6).

Lemma 2.1. Let $k \geq 1$ be an integer number. If $C_{k}(r)=\int_{a}^{b} \frac{\cos (k \theta)}{r^{2}-2 r \cos \theta+1} d \theta$ and $S_{k}(r)=$ $\int_{a}^{b} \frac{\sin (k \theta)}{r^{2}-2 r \cos \theta+1} d \theta$, for $r \neq 0$ and $r \neq 1$, then

$$
C_{k+1}(r)=\frac{\sin (k b)-\sin (k a)}{k r}+\frac{r^{2}+1}{r} C_{k}(r)-C_{k-1}(r)
$$

and

$$
S_{k+1}(r)=\frac{\cos (k b)-\cos (k a)}{k r}+\frac{r^{2}+1}{r} S_{k}(r)-S_{k-1}(r) .
$$


Proof. The recurrence (7), for the function $C_{k}$, follows doing the next computations:

$$
\begin{aligned}
\int_{a}^{b} \cos (k \theta) d \theta & =\int_{a}^{b} \cos (k \theta) \frac{r^{2}-2 r \cos \theta+1}{r^{2}-2 r \cos \theta+1} d \theta \\
& =\left(r^{2}+1\right) C_{k}(r)-2 r \int_{a}^{b} \frac{\cos (k \theta) \cos \theta}{r^{2}-2 r \cos \theta+1} d \theta \\
& =\left(r^{2}+1\right) C_{k}(r)-r \int_{a}^{b} \frac{\cos ((k+1) \theta)}{r^{2}-2 r \cos \theta+1} d \theta+r \int_{a}^{b} \frac{\cos ((k-1) \theta)}{r^{2}-2 r \cos \theta+1} d \theta \\
& =\left(r^{2}+1\right) C_{k}(r)-r C_{k+1}(r)-r C_{k-1}(r) .
\end{aligned}
$$

The recurrence (8), for $S_{k}$, follows similarly.

Lemma 2.2. Let $k \geq 0$ be an integer number. The functions defined in (6) satisfy the next relations:

(a) $C_{i, k}^{ \pm}(r)=\mp \frac{r^{k} \pi}{r^{2}-1}$ and $S_{i, k}^{ \pm}(r)=\frac{-p_{k-1}\left(r^{2}\right)}{r^{k}} L(r)+\frac{q_{i, k-2}^{ \pm}\left(r^{2}\right)}{r^{k-1}}$, when $0<r<1$,

(b) $C_{e, k}^{ \pm}(r)= \pm \frac{\pi}{r^{k}\left(r^{2}-1\right)}$ and $S_{e, k}^{ \pm}(r)=\frac{-p_{k-1}\left(r^{2}\right)}{r^{k}} L\left(\frac{1}{r}\right)+\frac{q_{e, k-2}^{ \pm}\left(r^{2}\right)}{r^{k-1}}$, when $r>1$,

where $L(r)=\log \left(\frac{1-r}{1+r}\right)$ is defined in the interval $(0,1)$. Moreover, $p_{k-1}\left(r^{2}\right)=\sum_{j=0}^{k-1} r^{2 j}$, for $k \geq 1, q_{j, k-2}^{ \pm}\left(r^{2}\right)$ are polynomials of degree $k-2$, for $k \geq 2$, and $j \in\{i, e\}$; and, for compactness, $p_{-1} \equiv 0$ and $q_{j,-2}^{ \pm} \equiv q_{j,-1}^{ \pm} \equiv 0$, for $j \in\{i, e\}$.

Proof. We prove only item (a). Item (b) follows similarly.

$\left(a_{1}\right)$ Straightforward computations show the cases $k=0,1$. Assuming the recurrence relation $(7)$ in the intervals $(0, \pi)$ and $(0,-\pi)$ we have that

$$
C_{i, k}^{ \pm}=\frac{\left(r^{2}+1\right)}{r}\left(\mp \frac{r^{k-1} \pi}{r^{2}-1}\right)-\left(\mp \frac{r^{k-2} \pi}{r^{2}-1}\right)=\mp \frac{r^{k} \pi}{r^{2}-1}
$$

$\left(a_{2}\right)$ As in the above case, straightforward computations show the cases $k=0,1$. From the recurrence (8), for $S_{i, k}^{ \pm}$, we have

$$
\begin{aligned}
\frac{p_{k-1}\left(r^{2}\right)}{r^{k}} L(r) & =\left(\frac{r^{2}+1}{r} \frac{p_{k-2}\left(r^{2}\right)}{r^{k-1}}-\frac{p_{k-3}\left(r^{2}\right)}{r^{k-2}}\right) L(r), \\
\frac{q_{k-2}\left(r^{2}\right)}{r^{k-1}} & =\frac{(-1)^{k-1}-1}{(k-1) r}+\frac{r^{2}+1}{r} \frac{q_{k-3}\left(r^{2}\right)}{r^{k-2}}-\frac{q_{k-4}\left(r^{2}\right)}{r^{k-3}} .
\end{aligned}
$$

The above expressions provide the next recurrence relations:

$$
\begin{aligned}
& p_{k-1}\left(r^{2}\right)=\left(r^{2}+1\right) p_{k-2}\left(r^{2}\right)-r^{2} p_{k-3}\left(r^{2}\right), \\
& q_{k-2}\left(r^{2}\right)=\frac{(-1)^{k-1}-1}{k-1} r^{k-2}+\left(r^{2}+1\right) q_{k-3}\left(r^{2}\right)-r^{2} q_{k-4}\left(r^{2}\right) .
\end{aligned}
$$

The proofs of the expressions of $S_{i, k}^{ \pm}$given in the statement follow by induction taking into account that, in the last expression, the term $r^{k-2}$ vanishes when $k$ is odd. We have not indicated the dependence of the polynomials $q_{k}$ in terms of the inner region, only that they are of degree $k$.

Proof of Theorem 1.2. We consider only $r \in(0,1)$. When $r>1$ the proof follows analogously. 
The inner Abelian integral (5), using Lemma 2.2 when $0<r<1$, writes as

$$
\begin{aligned}
I_{i}(r)= & \sum_{k=0}^{n+1} r^{k} R_{k}^{+}\left(r^{2}\right) C_{i, k}^{+}(r)+\sum_{k=1}^{n+1} r^{k} T_{k}^{+}\left(r^{2}\right) S_{i, k}^{+}(r) \\
& -\sum_{k=0}^{n+1} r^{k} R_{k}^{-}\left(r^{2}\right) C_{i, k}^{-}(r)-\sum_{k=1}^{n+1} r^{k} T_{k}^{-}\left(r^{2}\right) S_{i, k}^{-}(r) \\
= & \left(\sum_{k=1}^{n+1} r^{k} T_{k}^{+}\left(r^{2}\right) \frac{p_{k-1}\left(r^{2}\right)}{r^{k}}-\sum_{k=1}^{n+1} r^{k} T_{k}^{-}\left(r^{2}\right) \frac{p_{k-1}\left(r^{2}\right)}{r^{k}}\right) L(r) \\
& -\sum_{k=0}^{n+1} r^{k} R_{k}^{+}\left(r^{2}\right) \frac{r^{k} \pi}{r^{2}-1}+\sum_{k=1}^{n+1} r^{k} T_{k}^{+}\left(r^{2}\right) \frac{q_{i, k-2}^{+}\left(r^{2}\right)}{r^{k-1}} \\
& -\sum_{k=0}^{n+1} r^{k} R_{k}^{-}\left(r^{2}\right) \frac{r^{k} \pi}{r^{2}-1}-\sum_{k=1}^{n+1} r^{k} T_{k}^{-}\left(r^{2}\right) \frac{q_{i, k-2}^{-}\left(r^{2}\right)}{r^{k-1}} .
\end{aligned}
$$

Therefore we obtain that the coefficient of $L(r)$ is a polynomial of degree $n$. Because each term in the sum

$$
H_{n}\left(r^{2}\right)=\sum_{k=1}^{n+1} T_{k}^{+}\left(r^{2}\right) p_{k-1}\left(r^{2}\right)-\sum_{k=1}^{n+1} T_{k}^{-}\left(r^{2}\right) p_{k-1}\left(r^{2}\right),
$$

has degree $s_{k}=[n+1-k / 2]+k-1$, which is a nondecreasing sequence in $k$. So the greatest degree is achieved for $k=n+1$. Consequently $s_{n+1}=n$, as we wanted to prove. For the independent term we can argue similarly to prove that it has degree $2 n+1$. In particular, we can write

$$
\begin{aligned}
R_{2 n+1}(r)= & -\pi \sum_{k=0}^{n+1} r^{2 k-1} R_{k}^{+}\left(r^{2}\right)-\pi \sum_{k=0}^{n+1} r^{2 k-1} R_{k}^{-}\left(r^{2}\right) \\
& +\sum_{k=1}^{n+1}\left(r^{2}-1\right) T_{k}^{+}\left(r^{2}\right) q_{i, k-2}^{+}\left(r^{2}\right)-\sum_{k=1}^{n+1}\left(r^{2}-1\right) T_{k}^{-}\left(r^{2}\right) q_{i, k-2}^{-}\left(r^{2}\right) .
\end{aligned}
$$

Therefore, the highest degree term in each of the above sums is $t_{k}=[n+1-k / 2]+(2 k-$ $1) / 2$, which is also a nondecreasing sequence in $k$. So when $k=n+1$ we have that the degree of $R_{2 n+1}$ is $t_{n+1}=2 n+1$. This finishes the proof of the first part of the statement.

For the second part, we use the change of variables $r=(1-s) /(1+s)$ in $(4)$, for $0<r<1$. Now, the Abelian integral $I_{i}$ writes as

$$
\widetilde{I}_{i}(s)=\frac{\widetilde{R}_{2 n+4}(s)+\widetilde{H}_{2 n+3}(s) \log s}{4 s(1+s)^{2 n+3}},
$$

where $\widetilde{R}_{m}$ and $\widetilde{H}_{m}$ are polynomials of degree $m$. The proof finishes because the ordered family $\left\{1, \log s, s, s \log s, s^{2}, s^{2} \log s, \ldots\right\}$ is an ECT-system. Because the total terms in the numerator of $\widetilde{I}_{i}$ is $4 n+9=(2 n+4+1)+(2 n+3+1)$ and, consequently, it has $4 n+8$ simple zeros.

\section{Studying the REgions $\mathcal{R}_{i}$ AND $\mathcal{R}_{e}$ SEPARATELY}

This section is devoted to study the case $n=3$. With the change $r \rightarrow 1 / r$ in the outer region, the functions $I_{i}$ and $I_{e}$ share the interval of definition, that is $r \in(0,1)$. In fact, we will use $I_{e}(1 / r)=\widetilde{I}_{e}(r)$ for $r \in(0,1)$. The first part of Theorem 1.3 provides the number of zeros of the functions $I_{i}$ and $I_{e}$ separately. Its proof follows directly from the 
results of this section. In the first two we provide the expressions of $I_{i}$ and $I_{e}$ in terms of independent functions and which is the maximum number of simple zeros. As we will see, these functions are not an ECT-system. Consequently, we can not give a direct proof that the upper bounds are achieved. The last two explain which is the highest value for the multiplicity of a zero and how the maximum number of zeros varies when a zero of the highest multiplicity moves along the interval of definition.

Lemma 3.1. For $n=3$, the inner Abelian integral (4) can be written as

$$
I_{i}(r ; \beta)=\sum_{j=0}^{8} \beta_{j} f_{j}(r),
$$

where

$$
\begin{aligned}
& f_{0}(r)=\frac{r^{2}}{r^{2}-1}, \quad f_{1}(r)=\frac{r^{4}}{r^{2}-1}, \quad f_{2}(r)=\frac{r^{6}}{r^{2}-1}, \\
& f_{3}(r)=\frac{r^{8}}{r^{2}-1}, \quad f_{4}(r)=r, \quad f_{5}(r)=\frac{1}{2} r^{4} L(r)+r^{3}, \\
& f_{6}(r)=\frac{1}{6} r^{4}\left(3 r^{2}-1\right) L(r)+r^{5}, \quad f_{7}(r)=L(r), \\
& f_{8}(r)=r^{2} L(r), \quad L(r)=\log \left(\frac{1-r}{1+r}\right),
\end{aligned}
$$

and $\beta=\left(\beta_{0}, \ldots, \beta_{8}\right)$. For $r \in(0,1)$, the function $I_{i}(r ; \beta)$, has at most 9 zeros.

Proof. From the proof of Theorem 1.3, also using Lemma 2.2, the function $I_{i}$ can be written as (9), in fact it writes as a linear combination of 9 different functions $\left\{f_{0}, f_{1}, \ldots, f_{8}\right\}$. First we change the order of the functions to $\left[f_{0}, f_{1}, f_{2}, f_{3}, f_{4}, f_{6}, f_{5}, f_{7}, f_{8}\right]$. The proof follows showing that, with this new order, the first 8 Wronskian are non vanishing and the last has exactly one simple zero. Then, from [23], the statement is proved.

We show only why the last Wronskian has exactly one zero. The others follow similarly. Straightforward computations show that $W_{8}(r)=5218385264640\left(H_{0}(r) L(r)+\right.$ $\left.H_{1}(r)\right) /\left(r^{2}-1\right)^{25}$ with

$$
\begin{aligned}
& H_{0}(r)=15\left(3 r^{14}-13 r^{12}+63 r^{10}+63 r^{8}+553 r^{6}-231 r^{4}+1365 r^{2}+245\right)\left(r^{2}-1\right), \\
& H_{1}(r)=2 r\left(45 r^{14}-225 r^{12}+301 r^{10}+5495 r^{8}-7665 r^{6}+17605 r^{4}-18025 r^{2}-3675\right) .
\end{aligned}
$$

As $H_{0}(r) \neq 0$ in $(0,1)$ we can consider $\bar{W}_{8}(r)=W_{8}(r) / H_{0}(r)=L(r)+H_{1}(r) / H_{0}(r)$. Hence its first derivative is

$$
\bar{W}_{8}^{\prime}(r)=\frac{512 r^{8}\left(3 r^{10}-21 r^{8}+70 r^{6}-210 r^{4}-105 r^{2}+7\right)\left(5 r^{8}-28 r^{6}+70 r^{4}-140 r^{2}-35\right)}{5\left(r^{2}-1\right)^{2}\left(3 r^{14}-13 r^{12}+63 r^{10}+63 r^{8}+553 r^{6}-231 r^{4}+1365 r^{2}+245\right)^{2}} .
$$

Clearly $\bar{W}_{8}^{\prime}$ has only one zero in $(0,1)$. The proof finishes because the series of $W_{8}$ starts with a positive term and $\lim _{r \rightarrow 1^{-}} W_{8}(r)=-\infty$.

We remark that we have not reordered the functions in the statement of the above and next result because the crossed relation between the perturbed coefficients when we consider also the simultaneous bifurcation in next sections.

Lemma 3.2. For $n=3$ the outer Abelian integral (4), after the change $r \rightarrow 1 / r$, can be written as

$$
\widetilde{I}_{e}(r ; \gamma)=\sum_{j=0}^{7} \gamma_{j} g_{j}(r)
$$


where

$$
\begin{aligned}
g_{0}(r) & =\frac{r^{4}}{r^{2}-1}, & g_{1}(r) & =r^{5}, \\
g_{2}(r) & =\frac{1}{2} r^{2} L(r)+r^{3}, & g_{3}(r) & =\frac{1}{6}\left(3-r^{2}\right) L(r)+r, \\
g_{4}(r) & =r^{6} L(r), & g_{5}(r) & =r^{4} L(r), \\
g_{6}(r) & =r^{4}\left(r^{2}+1\right), & g_{7}(r) & =r^{4}, \quad L(r)=\log \left(\frac{1-r}{1+r}\right),
\end{aligned}
$$

and $\gamma=\left(\gamma_{0}, \ldots, \gamma_{7}\right)$. For $r \in(0,1)$, the function $\widetilde{I}_{e}(r ; \gamma)$ has at most 8 zeros.

Proof. We follow the same scheme as in Lemma 3.1 but for the function $\widetilde{I}_{e}(r)=I_{e}(1 / r)$ that can be obtained from (10). The ordered family to be considered now is $\left[g_{7} / r^{4}, g_{1} / r^{4}\right.$, $\left.\left(g_{6}-g_{7}\right) / r^{4}, g_{5} / r^{4}, g_{2} / r^{4}, g_{3} / r^{4}, g_{4} / r^{4}, g_{0} / r^{4}\right]$. We have divided all by $r^{4}$ to simplify the computations.

As in the previous proof, the first Wronskians are non vanishing but the last, $W_{7}$, has exactly one simple zero. Then, using [23], the statement follows.

Straightforward computations show that the first four Wronskians, $W_{0}, W_{1}, W_{2}$, and $W_{3}$, have no zeros and $W_{4}(r)=\left(H_{0}(r) L(r)+H_{1}(r)\right) /\left(r^{6}\left(r^{2}-1\right)^{6}\right)$ with

$$
\begin{aligned}
& H_{0}(r)=96\left(3 r^{4}-22 r^{2}-5\right)\left(r^{2}-1\right)^{2}, \\
& H_{1}(r)=-64 r\left(51 r^{6}-111 r^{4}+41 r^{2}+15\right) .
\end{aligned}
$$

As $H_{0}(r) \neq 0$ in $(0,1)$ and the first derivative of $\bar{W}_{4}(r)=L(r)+H_{1}(r) / H_{0}(r)$,

$$
\bar{W}_{4}^{\prime}(r)=\frac{8 r^{6}\left(3 r^{2}+1\right)\left(5 r^{4}+2 r^{2}+1\right)}{\left(r^{2}-1\right)^{3}\left(3 r^{4}-22 r^{2}-5\right)^{2}}
$$

does not vanish, we have that $W_{4}$ is non vanishing.

The next Wronskians need a more accurate analysis because they are polynomials of degree 2 in $L(r)$. We follow the same approach as in [22], that uses [13]. More concretely, the corresponding Wronskian writes as $W_{5}(r)=256\left(H_{0}(r)(L(r))^{2}+H_{1}(r) L(r)+\right.$ $\left.H_{2}(r)\right) /\left(r^{15}\left(r^{2}-1\right)^{9}\right)$ with

$$
\begin{aligned}
& H_{0}(r)=45\left(r^{2}-1\right)^{4}\left(9 r^{6}+55 r^{4}+203 r^{2}+35\right), \\
& H_{1}(r)=12 r\left(r^{2}-1\right)\left(10 r^{12}-422 r^{10}+3631 r^{8}-8767 r^{6}+7790 r^{4}-1645 r^{2}-525\right), \\
& H_{2}(r)=4 r^{2}\left(60 r^{12}+5983 r^{10}-25701 r^{8}+41052 r^{6}-26870 r^{4}+3885 r^{2}+1575\right),
\end{aligned}
$$

and $H_{0}(r)$ has no zeros in $(0,1)$. Then the solutions of $W_{5}(r)=0$ correspond with the intersections of the two curves $f(r, s)=0$ and $g(r, s)=0$ defined by

$$
\begin{aligned}
& f(r, s)=s-L(r), \\
& g(r, s)=H_{0}(r) s^{2}+H_{1}(r) s+H_{2}(r),
\end{aligned}
$$

in the region $r \in(0,1)$ and $s<0$. In fact we use the derivative of $g$ with respect to $f$, $h(r, s)=\frac{\partial g}{\partial s} \frac{2}{r^{2}-1}+\frac{\partial g}{\partial r}$, because we are considering the intersections of two algebraic curves. Straightforward computations show that the curves $g$ and $h$,

$$
\begin{aligned}
h(r, s)= & 18 r\left(\left(315 r^{12}-2730 r^{10}+11925 r^{8}-22860 r^{6}+19605 r^{4}-6570 r^{2}+315\right) s^{2}\right. \\
& +\left(100 r^{13}-3654 r^{11}+28902 r^{9}-70438 r^{7}+69786 r^{5}-25860 r^{3}+1260 r\right) s \\
& \left.+\left(200 r^{12}+15392 r^{10}-52272 r^{8}+61292 r^{6}-25440 r^{4}+1260 r^{2}\right)\right),
\end{aligned}
$$

have no intersection for $r \in(0,1)$. This is due to the fact that the resultant of both polynomials with respect to $s$,

$$
\begin{aligned}
\operatorname{Res}(g, h, s)= & 59719680 r^{30}\left(r^{2}-1\right)^{4}\left(30 r^{6}+23 r^{4}+16 r^{2}+3\right) \\
& \left(350 r^{10}+295 r^{8}+240 r^{6}+194 r^{4}+58 r^{2}+15\right),
\end{aligned}
$$


never vanishes for $r \in(0,1)$. The curves $f$ and $g$ coincide at $(0,0)$, where they are tangent. Thus, using the generalized Rolle's Theorem for curves, see [13], we have proved that $f$ and $g$ have no intersection points with $r \in(0,1)$. Consequently, $W_{5}(r) \neq 0$ in $r \in(0,1)$.

The proof that $W_{6}$ is non vanishing follows similarly. In this case, the nonintersection property, except the tangent point at $(0,0)$, is even simpler to prove. Because the corresponding resultant, $\operatorname{Res}(g, h, s)$, is $r^{38}\left(1-r^{2}\right)^{2}$, modulus a multiplicative constant.

Finally, it remains only the study of the last Wronskian, that needs a more accurate analysis. It writes as $W_{7}=-84934656\left(H_{0}(r)(L(r))^{2}+H_{1}(r) L(r)+H_{2}(r)\right) /\left(r^{19}\left(r^{2}-1\right)^{20}\right)$ with

$$
\begin{aligned}
H_{0}(r)= & 15\left(r^{2}-1\right)^{2}\left(35 r^{14}-315 r^{12}+651 r^{10}-6523 r^{8}+18193 r^{6}\right. \\
& \left.-12201 r^{4}-10815 r^{2}+735\right), \\
H_{1}(r)= & 4 r\left(40 r^{18}-1475 r^{16}+9680 r^{14}-64320 r^{12}+301680 r^{10}\right. \\
& \left.-662534 r^{8}+492240 r^{6}+93240 r^{4}-180600 r^{2}+11025\right), \\
H_{2}(r)= & 4 r^{2}\left(80 r^{16}+2013 r^{14}-36257 r^{12}+174713 r^{10}-504557 r^{8}\right. \\
& \left.+488775 r^{6}+35245 r^{4}-176925 r^{2}+11025\right) .
\end{aligned}
$$

Using the same procedure as in the previous cases we have that the corresponding function $h(r, s)=\frac{r^{2}-1}{2 r}\left(\frac{\partial g}{\partial s} \frac{2}{r^{2}-1}+\frac{\partial g}{\partial r}\right)$ writes as

$$
\begin{aligned}
h(r, s)= & 15\left(1-r^{2}\right)^{2}\left(315 r^{14}-2765 r^{12}+6447 r^{10}-42393 r^{8}+117057 r^{6}\right. \\
& \left.-103383 r^{4}-8043 r^{2}+12285\right) s^{2}-4 r\left(1-r^{2}\right)\left(380 r^{16}-12275 r^{14}+69975 r^{12}\right. \\
& \left.-410835 r^{10}+1605435 r^{8}-2796033 r^{6}+1494885 r^{4}+243495 r^{2}-184275\right) s \\
& +4 r^{2}\left(760 r^{16}+13909 r^{14}-260223 r^{12}+1237757 r^{10}-3269383 r^{8}+3815351 r^{6}\right. \\
& \left.-1357125 r^{4}-366345 r^{2}+184275\right)
\end{aligned}
$$

and the resultant as

$$
\begin{aligned}
\operatorname{Res}(g, h, s)= & 5033164800 r^{36}\left(r^{2}-1\right)^{2}\left(5 r^{14}-40 r^{12}+135 r^{10}+782 r^{8}+243 r^{6}-132 r^{4}\right. \\
& \left.+33 r^{2}-2\right)\left(35 r^{18}-350 r^{16}+1540 r^{14}+12656 r^{12}+5346 r^{10}-4620 r^{8}\right. \\
& \left.+2340 r^{6}-640 r^{4}+83 r^{2}-6\right) .
\end{aligned}
$$

But now $H_{0}$ has a zero, $r_{1} \approx 0.25258$, and $\operatorname{Res}(g, h, s)$ has two, $r_{2} \approx 0.28749$ and $r_{3} \approx$ 0.47708. The algebraic system, $\{g=0, h=0\}$, defined in (11) and (12), of degree 2 in $s$ can be rewritten, using Groebner basis, as an equivalent one of degree 1 in $s$. This new system has only two intersection points $\left(r_{2}, s_{2}\right)$ and $\left(r_{3}, s_{3}\right)$, with $s_{2} \approx-0.59167$ and $s_{3} \approx-1.03840$, in the region where we are interested, $r \in(0,1)$ and $s<0$. Moreover solving $W_{7}=0$ with respect to $L$, we can write both solutions as

$$
L_{ \pm}=\frac{-H_{1} \pm \sqrt{H_{1}^{2}-4 H_{0} H_{2}}}{2 H_{0}} .
$$

Notice that in $r_{1}$, one of the roots has an asymptote and the other not. This is due to the fact that $H_{0}\left(r_{1}\right)=0, H_{0}^{\prime}\left(r_{1}\right) \neq 0, H_{1}\left(r_{1}\right) \neq 0$ and $H_{2}\left(r_{1}\right) \neq 0$. Furthermore, $H_{0}=\left(r-r_{1}\right) \widetilde{H}_{0}$ and $H_{0}^{\prime}=\widetilde{H}_{0}+\left(r-r_{1}\right) \widetilde{H}_{0}^{\prime}$, that is $H_{0}^{\prime}\left(r_{1}\right)=\widetilde{H}_{0}\left(r_{1}\right)$. From these conditions we have the first terms of the series expansion of $L_{ \pm}$at $r_{1}$ :

$$
\begin{aligned}
L_{+} & =-\frac{H_{1}\left(r_{1}\right)}{\widetilde{H}_{0}\left(r_{1}\right)\left(r-r_{1}\right)}+\cdots=-\frac{H_{1}\left(r_{1}\right)}{H_{0}^{\prime}\left(r_{1}\right)\left(r-r_{1}\right)}+\cdots, \\
L_{-} & =-\frac{H_{2}\left(r_{1}\right)}{H_{1}\left(r_{1}\right)}+\cdots .
\end{aligned}
$$


Additionally, the function $L$, in the interval $(0,1 / 2)$, is between the next two functions:

$$
\begin{aligned}
M_{ \pm}= & -2 r-\frac{2}{3} r^{3}-\frac{2}{5} r^{5}-\frac{2}{7} r^{7}-\frac{2}{9} r^{9}-\frac{2}{11} r^{11}-\frac{2}{13} r^{13}-\frac{2}{15} r^{15}-\frac{2}{17} r^{17} \\
& -\frac{2}{19} r^{19}-\frac{2}{21} r^{21}-\frac{2}{23} r^{23}-\frac{2}{25} r^{25} \pm \frac{1}{4} r^{27} .
\end{aligned}
$$

This last property follows studying the series expansion of $L$ in the neighborhood of the origin. In fact, $M_{+}-L=35 r^{27} / 108+O\left(r^{29}\right)$ and $L-M_{-}=19 r^{27} / 108+O\left(r^{29}\right)$. Thus $\mathrm{d}\left(M_{+}-L\right) / \mathrm{d} r$ and $\mathrm{d}\left(L-M_{-}\right) / \mathrm{d} r$ does not have zeros in $(0,1 / 2)$. Now replacing $M_{ \pm}$in $W_{7}$ instead of $L$, we have that $W_{7}\left(M_{ \pm}\right)$in the interval $(0,1 / 2)$ is non vanishing. Moreover, in the neighborhood of $r=0$ we have $L-L_{+}=-704 r^{9} / 11025+\cdots$ and $L-L_{-}=$ $1024 r^{13} / 7630623+\cdots$. And, in the neighborhood of $r=1$, we have $L_{-}(1)=-23 / 4$, and $L-L_{+}=1 /(r-1)^{2}-\log ((1-r) / 2)+\cdots$.

We finish the proof applying Rolle's Theorem separately in the intervals $\left(0, r_{1}\right)$ and $\left(r_{1}, 1\right)$, where the functions $L$ and $L_{ \pm}$are well defined and smooth. As the tangency points satisfy $r_{2}<1 / 2$ and $r_{3}<1 / 2$, we can conclude that only one intersection point can exist and it is between $L$ and $L_{-}$. So $W_{7}$ vanishes at most once. All these properties can be seen in Figure 3 .

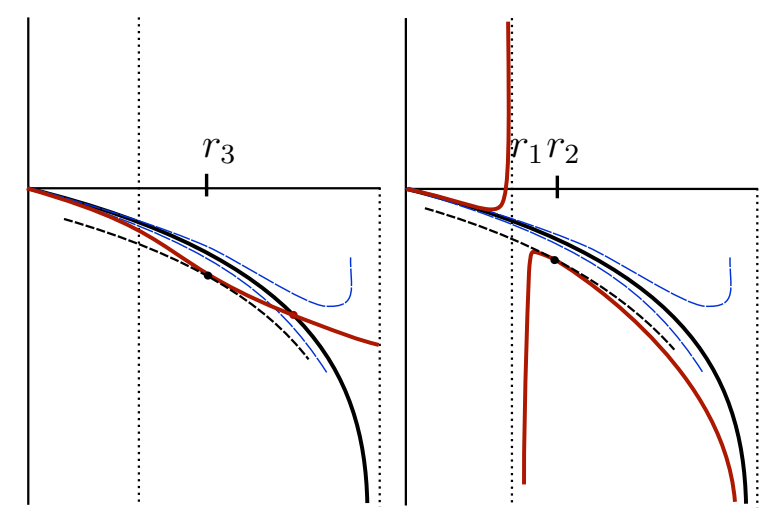

Figure 3. Graphs of $L$ (in black), $L_{+}(r)$ (in red on the left), $L_{-}(r)$ (in red on the right) and $M_{ \pm}(r)$ (in blue) on $(0,1)$. The values $r_{1}, r_{2}$ and $r_{3}$ are depicted also.

Remark 3.3. In the above proof, as the resultant (13) vanishes three times, the results of [23] provide a worst upper bound than if an accurate analysis is done. The main difficulty has been to provide an analytic argument showing which are the tangent points providing intersection points and which not.

Following also the ideas of [23], and because we will need in the following section, the next two results study which are the maximal multiplicity zeros of the inner and outer Abelian integrals (3) and the corresponding unfolding of simple zeros bifurcating from them.

Proposition 3.4. (a) Let $\rho^{o} \approx 0.27055$ be the zero in $(0,1)$ of the function

$$
\begin{aligned}
w_{i, 8}(r)= & 15\left(1-r^{2}\right)\left(3 r^{14}-13 r^{12}+63 r^{10}+63 r^{8}+553 r^{6}-231 r^{4}+1365 r^{2}+245\right) L(r) \\
& -2 r\left(45 r^{14}-225 r^{12}+301 r^{10}+5495 r^{8}-7665 r^{6}+17605 r^{4}-18025 r^{2}-3675\right) .
\end{aligned}
$$

Let $\hat{\beta}$ be a point in the parameters space such that the function $I_{i}(r ; \hat{\beta})$, defined in (9), has a zero of multiplicity $k$ at $\rho$. Then $1 \leq k \leq 8$ for $\rho \neq \rho^{o}$ and $k=9$ when $\rho=\rho^{o}$. 
Moreover, for these values of $k$, there exist $\beta$ in a neighborhood of $\hat{\beta}$ such that $I_{i}(r ; \beta)$ has $k$ simple zeros in a small enough neighborhood of $\rho^{o}$.

(b) Let $\eta^{o} \approx 0.5143$ be the zero in $(0,1)$ of the function

$$
\begin{aligned}
w_{e, 7}(r)= & 15\left(1-r^{2}\right)^{2}\left(35 r^{14}-315 r^{12}+651 r^{10}-6523 r^{8}+18193 r^{6}-12201 r^{4}\right. \\
& \left.-10815 r^{2}+735\right)(L(r))^{2}+4 r\left(40 r^{18}-1475 r^{16}+9680 r^{14}-64320 r^{12}\right. \\
& \left.+301680 r^{10}-662534 r^{8}+492240 r^{6}+93240 r^{4}-180600 r^{2}+11025\right) L(r) \\
& +4 r^{2}\left(80 r^{16}+2013 r^{14}-36257 r^{12}+174713 r^{10}-504557 r^{8}+488775 r^{6}\right. \\
& \left.+35245 r^{4}-176925 r^{2}+11025\right) .
\end{aligned}
$$

Let $\tilde{\beta}$ be a point in the parameters space such that the function $\widetilde{I}_{e}(r ; \tilde{\beta})$, defined in (10), has a zero of multiplicity $\ell$ at $\eta$. Then $1 \leq \ell \leq 7$ for $\eta \neq \eta^{\circ}$ and $\ell=8$ when $\eta=\eta^{\circ}$. Moreover, for these values of $\ell$, there exist $\beta$ in a neighborhood of $\tilde{\beta}$ such that $\widetilde{I}_{e}(r ; \beta)$ has $\ell$ simple zeros in a small enough neighborhood of $\eta^{\circ}$.

Proof. We prove only the maximal multiplicity cases for $I_{i}$, that are $k=8,9$. The other cases follow similarly.

First we show how the parameters $\beta_{j}$, for $j=0, \ldots, 7$, of the function $I_{i}$ can be written in terms of $\rho$, when $\rho$ is a zero of multiplicity eight of it. Hence, this function writes only using $r, \rho, L(r), L(\rho)$. We recall that $0<r<1$ and, consequently, $0<\rho<1$.

Assume that $\rho$ is a root of multiplicity eight. We consider the linear system of 8 equations and 8 variables $\beta_{j}, j=0, \ldots, 7$, defined by $\frac{d^{j} I_{i}}{d r^{j}}(\rho)=0$ for $j=0, \ldots, 7$. As the Wronskian defined by $\left[f_{0}, f_{1}, \ldots, f_{7}\right]$ does not vanish for $\rho \in(0,1)$, the variables $\beta_{j}$, $j=0, \ldots, 7$, can be written as $\beta_{j}=\hat{f}_{j}(\rho) \beta_{8}$, with $\hat{f}_{j}(\rho)$ functions of $\rho$ and $L(\rho)$. See $(9)$ for the definition of the functions $f_{j}$. Then, see for instance [23], the eighth derivative of $I_{i}$ at $\rho$ is

$$
\frac{d^{8} I_{i}}{d r^{8}}(\rho)=\left.\frac{W\left(\left[f_{0}, f_{1}, f_{2}, f_{3}, f_{4}, f_{6}, f_{5}, f_{7}, f_{8}\right], r\right)}{W\left(\left[f_{0}, f_{1}, f_{2}, f_{3}, f_{4}, f_{6}, f_{5}, f_{7}\right], r\right)}\right|_{r=\rho},
$$

where $W$ denotes the Wronskian function. In particular

$$
\left.W\left(\left[f_{0}, f_{1}, f_{2}, f_{3}, f_{4}, f_{6}, f_{5}, f_{7}, f_{8}\right], r\right)\right|_{r=\rho}=-5218385264640 w_{i, 8}(\rho) /\left(1-\rho^{2}\right)^{25},
$$

where $w_{i, 8}(\rho)$ is defined in (14). When $\rho \neq \rho^{o}$ clearly the multiplicity is eight and it can be checked that when $\rho=\rho^{o}$ the multiplicity is nine.

Finally, the unfolding of zeros of any perturbation (moving the parameters $\beta_{j}$ ) of $I_{i}$, in a small neighborhood of $r=\rho$, follows from the ECT-system (ECT-system with accuracy one) property for $\rho \neq \rho^{o}\left(\rho=\rho^{o}\right)$, see [23]. Consequently, the statement follows.

Proposition 3.5. (a) Let $\rho^{*} \approx 0.3029$ be the positive solution of

$$
\begin{aligned}
& 3\left(\rho^{12}-75 \rho^{8}+200 \rho^{6}-645 \rho^{4}+600 \rho^{2}+175\right) L(\rho) \\
& +2 \rho\left(3 \rho^{10}-95 \rho^{8}+390 \rho^{6}-1230 \rho^{4}+1975 \rho^{2}+525\right)=0 .
\end{aligned}
$$

Let $\hat{\beta}$ be a point in the parameters space such that $I_{i}$, defined in (9), has a zero of multiplicity eight at $\rho$. The maximum number of zeros of $I_{i}(r ; \beta)$ in $(0,1)$ is 9 or 8 if $\rho \in\left(0, \rho^{*}\right)$ or $\rho \in\left(\rho^{*}, 1\right)$, respectively, for all $\beta$ in a small neighborhood of $\hat{\beta}$. Moreover, these maximal numbers are achieved as simple ones. 
(b) Let $\eta^{*} \approx 0.57704$ be the positive solution of

$$
\begin{aligned}
& -135\left(1-\eta^{2}\right)^{4}\left(35 \eta^{10}-147 \eta^{8}+174 \eta^{6}+378 \eta^{4}+735 \eta^{2}+105\right)(L(\eta))^{3}-9 \eta\left(1-\eta^{2}\right) \\
& \left(5 \eta^{18}-615 \eta^{16}+6615 \eta^{14}-25425 \eta^{12}+25305 \eta^{10}-22047 \eta^{8}+91605 \eta^{6}-123795 \eta^{4}\right. \\
& \left.+40950 \eta^{2}+9450\right)(L(\eta))^{2}+12 \eta^{2}\left(1-\eta^{2}\right)\left(177 \eta^{16}-1136 \eta^{14}+6090 \eta^{12}+5226 \eta^{10}\right. \\
& \left.+18762 \eta^{8}-126900 \eta^{6}+165690 \eta^{4}-66150 \eta^{2}-14175\right) L(\eta)-12 \eta^{3}\left(369 \eta^{16}-4358 \eta^{14}\right. \\
& \left.+28517 \eta^{12}-30268 \eta^{10}-76323 \eta^{8}+178010 \eta^{6}-143325 \eta^{4}+37800 \eta^{2}+9450\right)=0 .
\end{aligned}
$$

Let $\hat{\gamma}$ be a point in the parameters space such that $\widetilde{I}_{e}$, defined in (10), has a zero of multiplicity seven at $\eta$. The maximum number of zeros of $\widetilde{I}_{e}(r ; \gamma)$ in $(0,1)$ is 8 or 7 if $\eta \in\left(0, \eta^{*}\right)$ or $\eta \in\left(\eta^{*}, 1\right)$, respectively, for all $\gamma$ in a small neighborhood of $\hat{\gamma}$. Moreover, these maximal numbers are achieved as simple ones.

Proof. We prove only the statement (a), the other case follows similarly.

As nine is the upper bound of the number of zeros of $I_{i}$, see Lemma 3.1, the distribution and the number of zeros, in terms of $\rho$, can be studied from the graph of $I_{i}$ in the full interval $(0,1)$. In fact, it only depends on the local plot near $r=0, r=\rho$, and $r=1$. We start studying how is this local behavior in terms of $\rho$ and we finish drawing the graph of the function $I_{i}$.

For the local behavior, first we consider $\rho \neq \rho^{o}$, see Proposition 3.4. Consequently $r=\rho$ is a zero of multiplicity 8 ( of $I_{i}$ ) for $\beta=\hat{\beta}$. Also from the proof of Proposition 3.4 we can assume that $\beta_{8}=1$ and, consequently, $I_{i}$ writes as a rational function in $\{r, \rho, L(r), L(\rho)\}$. The series expansions are denoted by $I_{i, 0}, I_{i, \rho}$, and $I_{i, 1}$, respectively. Straightforward computations show that

$$
\begin{aligned}
I_{i, 0} & =h_{i, 0}(\rho) r+O\left(r^{2}\right), \\
I_{i, \rho} & =h_{i, \rho}(\rho)(r-\rho)^{8}+O\left((r-\rho)^{9}\right), \\
I_{i, 1} & =h_{i, 1}(\rho) \frac{1}{1-r}+O\left(r^{0}\right),
\end{aligned}
$$

where $h_{i, \xi}=N_{i, \xi} / D_{i, \xi}$ for $\xi \in\{0, \rho, 1\}$ and

$$
N_{i, \xi}(\rho)=p_{0}^{\xi}(\rho)+p_{1}^{\xi}(\rho) L(\rho), \quad D_{i, \xi}(\rho)=q_{0}^{\xi}(\rho)+q_{1}^{\xi}(\rho) L(\rho),
$$

for $\xi \in\{0, \rho, 1\}$, with $p_{j}^{\xi}, q_{j}^{\xi}$ given polynomials. In fact, the denominators are related by the expressions $D_{i, \rho}(\rho)=7 \rho\left(\rho^{2}-1\right)^{5} D_{i, 0}(\rho), D_{i, 1}(\rho)=\rho D_{i, 0}(\rho) / 5$, and

$$
\begin{aligned}
D_{i, 0}(\rho) & =225\left(3 \rho^{14}-3 \rho^{12}-165 \rho^{10}+77 \rho^{8}-1071 \rho^{6}-609 \rho^{4}-175 \rho^{2}-105\right)\left(\rho^{2}-1\right)^{2} L(\rho) \\
& +30 \rho\left(45 \rho^{16}-960 \rho^{14}+3480 \rho^{12}-13248 \rho^{10}+23170 \rho^{8}-2240 \rho^{6}-5600 \rho^{4}-1575\right) .
\end{aligned}
$$

We remark that the three denominators do not vanish for $\rho \in(0,1)$. Now, we can draw $h_{i, 0}$, first studying the local behavior near the endpoints of the interval of definition and second studying when it vanishes. The series of $h_{i, 0}$ at $\rho=0$ is

$$
h_{i, 0}(\rho)=\frac{512}{4725} \rho^{8}-\frac{985088}{779625} \rho^{10}+\frac{140819456}{152026875} \rho^{12}+O\left(\rho^{14}\right)
$$

and $\lim _{\rho \rightarrow 1^{-}} h_{i, 0}(\rho)=-\infty$. When $\rho \in(0,1)$ the zeros of $h_{i, 0}(\rho)$ are obtained solving equation (16). Hence, as the coefficient of $L$ in (16) does not vanish, the number of zeros of it is given by the zeros of

$$
\Lambda(\rho)=L(\rho)+\frac{2}{3} \frac{\rho\left(3 \rho^{10}-95 \rho^{8}+390 \rho^{6}-1230 \rho^{4}+1975 \rho^{2}+525\right)}{\rho^{12}-75 \rho^{8}+200 \rho^{6}-645 \rho^{4}+600 \rho^{2}+175} .
$$


As $L^{\prime}(\rho)$ only has a zero, the drawing of $h_{i, 0}$ in $(0,1)$ is sketched in Figure 4. Clearly, equation (16) only has one positive zero which defines the bifurcation point $\rho^{*} \approx 0.3029$.

From (15) we know that $h_{i, \rho}=8 !\left(\mathrm{d}^{8} I_{i} / \mathrm{d} r^{8}\right)(\rho)$ that only has a positive zero at $\rho^{o}$, see equation (14). Consequently the drawing of $h_{i, \rho}$ in $(0,1)$ is sketched in Figure 4 because $\lim _{\rho \rightarrow 1^{-}} h_{i, \rho}(\rho)=-\infty$ and it series expansion at $\rho=0$ is

$$
h_{i, \rho}(\rho)=\frac{512}{4725} \rho-\frac{118784}{111375} \rho^{3}-\frac{714041344}{152026875} \rho^{5}+O\left(\rho^{7}\right) .
$$

Arguing as in the above cases and using that $\lim _{\rho \rightarrow 1^{-}} h_{i, 1}(\rho)=0$ and

$$
h_{i, 1}(\rho)=-\frac{2048}{4725} \rho+\frac{2220032}{779625} \rho^{3}-\frac{1308176384}{152026875} \rho^{5}+O\left(\rho^{7}\right),
$$

the drawing of $h_{i, 1}$ is sketched in Figure 4.
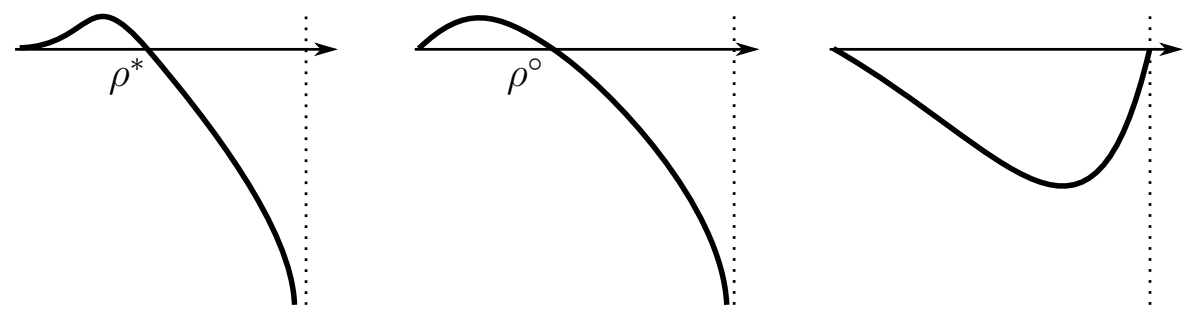

FigURE 4 . Graph of $h_{i, 0}(\rho), h_{i, \rho}(\rho)$, and $h_{i, 1}(\rho)$ on $(0,1)$, respectively

From the above description, it is clear that the graph of $I_{i}(r, \hat{\beta})$ for $r \in(0,1)$ depends on $\rho$. In particular, it can be seen that, when $\rho \in\left(0, \rho^{*}\right)$ and $\rho \neq \rho^{o}, I_{i}$ has a zero of multiplicity eight at $r=\rho$ and an extra simple zero in $(0,1)$. Moreover, when $\rho=\rho^{*} I_{i}$ has a zero of multiplicity nine and when $\rho \in\left(\rho^{*}, 1\right), I_{i}$ has no other zeros except the zero of multiplicity eight at $r=\rho$. The different plots of $I_{i}(r, \hat{\beta})$ for $r \in(0,1)$ when $\rho$ varies in $(0,1)$ can be seen in Figure 5 .

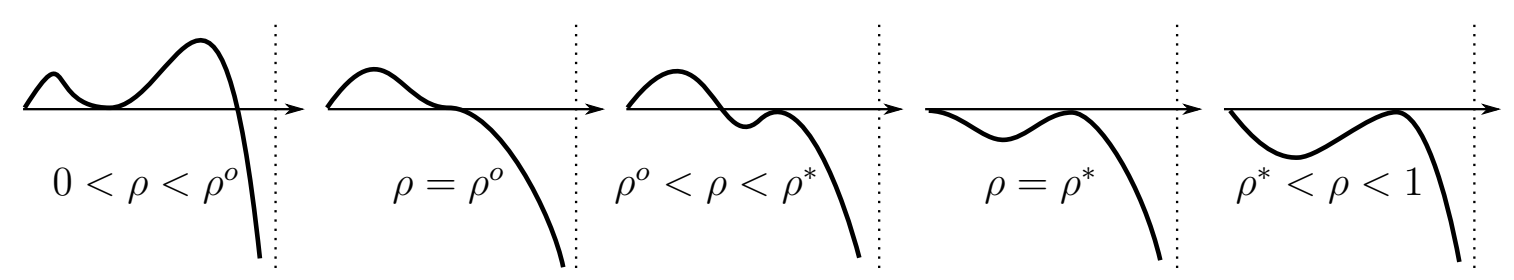

FiguRE 5. Graphs of $I_{i}(r, \hat{\beta})$ for $r \in(0,1)$ for different values of $\rho$.

Finally, the proof follows, also using also Proposition 3.4, choosing values of $\beta$ close enough to $\hat{\beta}$.

\section{Existence of simultaneous zeros in the Regions $\mathcal{R}_{i}$ and $\mathcal{R}_{e}$}

This section is devoted to prove the second part of Theorem 1.3, which provides a partial result about the simultaneous bifurcation of limit cycles in the inner and outer period annuli regions. Following the change of variable in the outer period annuli, $r \rightarrow 1 / r$, introduced in Lemma 3.2, we study the simultaneous zeros of the functions $I_{i}$ and $\widetilde{I}_{e}$, see (9) and (10). In Section 3, we have proved that the maximum number of zeros, separately, of the inner and outer Abelian integrals, is 9 and 8, respectively. Moreover, except for some special values of the perturbation parameters, the maximum multiplicity 
of each zero is 8 and 7, respectively. We remark that, while the number of parameters in the inner and outer annuli regions is 9 and 8, see Lemmas 3.1 and 3.2, we will see, in Lemma 4.1, that the total number of independent parameters is 11 . But the dimension of the essential parameter space is 10 , because it is not restrictive to assume that one of them is different from zero. A complete study of all possible configurations of zeros of both Abelian integrals is very difficult due to the high dimension of the parameter space. For this reason we study some special zones in this 10-dimensional space. More concretely, we will study all possible configurations of simultaneous zeros near zeros of multiplicities $(k, \ell)$ such that $k+\ell=10$, that is $(8,2),(7,3),(6,4),(5,5),(4,6)$, and $(3,7)$, see Propositions 4.2, 4.3, 4.4, 4.5, 4.6, and 4.7, respectively. Theorem 1.3 follows from them.

Straightforward computations show, see the next result, the relation between the parameters that appear in the definition of the inner and outer Abelian integrals, when we study the configuration of simultaneous zeros.

Lemma 4.1. When the zeros of the functions $I_{i}$ and $\widetilde{I}_{e}$ are considered simultaneously, the parameters $\beta$ and $\gamma$, defined in (9) and (10), can be written as $\beta_{j}=\alpha_{j}$ for $j=0, \ldots, 8$ and $\gamma_{0}=\alpha_{0}+\alpha_{1}+\alpha_{2}+\alpha_{3}$, and $\gamma_{j}=\alpha_{j+3}$ for $j=1, \ldots, 7$.

Our interest in this section will be not only the study of the number of zeros that have the functions $I_{i}$ and $\widetilde{I}_{e}$ simultaneously. We deal also with a partial study of the bifurcation diagram of them. As we have mentioned above, this is done by taking two simultaneous zeros $(\rho, \eta)$ with multiplicities $(k, \ell)$, in the inner and outer regions, such that $k+\ell=10$. Hence, as in the previous section, every point $(\rho, \eta) \in(0,1)^{2}$ corresponds with a line of points $\alpha=\left(\alpha_{0}, \ldots, \alpha_{10}\right) \in \mathbb{R}^{11}$. In some sense, we have compactified the parameter space transforming the 11-dimensional space to a compact region of a 2-dimensional space, in fact the unit square. The following results provide the different regions in $(0,1)^{2}$ corresponding with all possible configurations of simultaneous zeros of the functions $I_{i}$ and $\widetilde{I}_{e}$ in the full interval $(0,1)$ in a neighborhood of the full line defined by $\alpha$.

Proposition 4.2. Let $\rho, \eta$ be zeros of multiplicity 8 and 2 of $I_{i}$ and $\widetilde{I}_{e}$, respectively. There exist two curves $\Upsilon=\left\{\rho=\rho^{*}\right\}$ and $\Psi=\left\{N_{e, 0}(\rho, \eta)=0\right\}$ such that the square $(0,1)^{2}$ is divided in four regions, see Figure 6. In particular, $\rho^{*}$ is the unique zero of (16) and the function $N_{0}^{e}(\rho, \eta)$ writes as

$$
N_{e, 0}(\rho, \eta)=p_{0}^{0}+p_{1}^{0} L(\rho)+p_{2}^{0} L(\eta)+p_{3}^{0} L(\rho) L(\eta),
$$

with $p_{0}^{0}, p_{1}^{0}, p_{2}^{0}$, and $p_{3}^{0}$ polynomials in $\rho$ and $\eta$ of degrees $29,28,30$, and 29 , respectively. Then, the configurations of simple zeros of $I_{i}$ and $\widetilde{I}_{e}$, in a neighborhood of $(\rho, \eta)$, are $(9,3)$, $(9,2),(8,3)$, and $(8,2)$, respectively. Moreover, each of them is realizable only in one of the four regions.

Proof. The proof follows similarly as the proof of Proposition 3.4 studying the different plots of the functions $I_{i}(r)$ and $\widetilde{I}_{e}(r)$ in $r \in(0,1)$, but in terms of the parameters $\rho$ and $\eta$. We only show the main differences.

Let $\rho$ and $\eta$ be zeros of multiplicity 8 and 2 of the functions $I_{i}$ and $\widetilde{I}_{e}$, respectively, defined in Lemma 4.1. Straightforward computations provide the coefficients $\alpha_{j}$, for $j=0, \ldots, 10$, in terms of $\rho$ and $\eta$. We denote this special value as $\hat{\alpha}$. In fact, the function $I_{i}(r, \alpha(\rho))$ coincides with the obtained in Propositions 3.4 and 3.5. So, $\alpha_{j}=\beta_{j}$, for $j=0, \ldots, 7$, and $I_{i}$ is a function of $\{r, \rho, L(r), L(\rho)\}$ with $0<r, \rho<1$. Moreover the other components $\alpha_{8}, \alpha_{9}$, and $\alpha_{10}$ are functions of $\rho$ and $\eta$. Consequently, the function $\widetilde{I}_{e}$ writes in terms of $\{r, \rho, \eta, L(r), L(\rho), L(\eta)\}$ with $0<r, \rho, \eta<1$. 


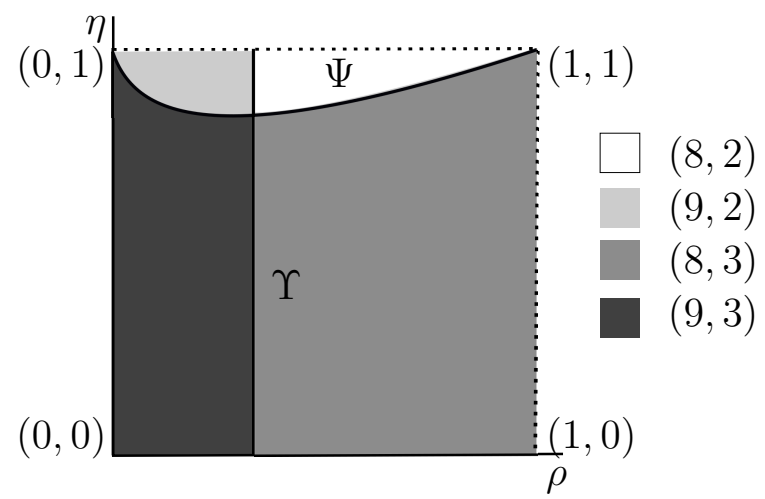

FiguRE 6. Bifurcation diagram of configuration of simultaneous zeros for the maximum multiplicity case $(8,2)$

The local and global studies for the function $I_{i}$ are, in fact, the same. Consequently, only remains the study of $\widetilde{I}_{e}$. Its series expansions at $r=0$, at $r=\eta$ and $r=1$ write as

$$
\begin{aligned}
& \widetilde{I}_{e, 0}=h_{e, 0}(\rho, \eta) r^{4}+O\left(r^{5}\right), \\
& \widetilde{I}_{e, \eta}=h_{e, \eta}(\rho, \eta)(r-\eta)^{2}+O\left((r-\eta)^{3}\right), \\
& \widetilde{I}_{e, 1}=h_{e, 1}(\rho, \eta) \frac{1}{1-r}+O(1),
\end{aligned}
$$

respectively. For each $\xi \in\{0, \eta, 1\}$, we denote the numerators and denominators by $N_{e, \xi}(\rho, \eta)=\operatorname{num}\left(h_{e, \xi}\right)$ and $D_{e, \xi}(\rho, \eta)=\operatorname{den}\left(h_{e, \xi}\right)$. As $D_{e, 0}=D_{e, 1}, D_{e, \eta}=D_{e, 1} /\left(\eta^{2}-1\right)$ and $N_{e, 1}<0$ with $0<\rho, \eta<1$, essentially, there are only three different functions to be studied:

$$
\begin{aligned}
& N_{e, 0}(\rho, \eta)=p_{0}^{0}+p_{1}^{0} L(\rho)+p_{2}^{0} L(\eta)+p_{3}^{0} L(\rho) L(\eta), \\
& N_{e, \eta}(\rho, \eta)=p_{0}^{\eta}+p_{1}^{\eta} L(\rho)+p_{2}^{\eta} L(\eta)+p_{3}^{\eta} L(\rho) L(\eta), \\
& D_{e, 1}(\rho, \eta)=q_{0}^{1}+q_{1}^{1} L(\rho)+q_{2}^{1} L(\eta)+q_{3}^{1} L(\rho) L(\eta),
\end{aligned}
$$

with $p_{0}^{0}, p_{1}^{0}, p_{2}^{0}$ and $p_{3}^{0}$ polynomials in $\rho$ and $\eta$ of degrees $29,30,28$, and $29 ; p_{0}^{\eta}, p_{1}^{\eta}, p_{2}^{\eta}$ and $p_{3}^{\eta}$ polynomials in $\rho$ and $\eta$ of degrees 33, 34, 30, and 31; and $q_{0}^{1}, q_{1}^{1}, q_{2}^{1}$ and $q_{3}^{1}$ polynomials in $\rho$ and $\eta$ with rational coefficients of degrees 29, 30, 28, and 29. We do not write here the explicit expressions of that polynomials because of the size of them.

The signs of the functions (17) define the topologically different plots of $\widetilde{I}_{e}(r, \hat{\alpha})$. The zero level curves of $N_{e, 0}, N_{e, \eta}$ and $D_{e, 1}$ are depicted in Figure 7 as black, red and blue dots lines, respectively. Moreover, they define six different regions denoted by $I, I I, I I I$, $I V, V$, and $V I$ in Figure 7. In Section 5 we do a more detailed study of that zero level curves near the corners of the domain of definition.

Now, analyzing the sign of the functions (17), it can be shown that in regions $I, I I$, $I I I$, and $I V$ the function $\widetilde{I}_{e}(r, \hat{\alpha})$ has a point of multiplicity 2 at $r=\eta$ and an extra simple zero in $(0,1)$. Moreover in regions $V$ and $V I$ only a zero of multiplicity 2 exits at $r=\eta$. Examples of both situations are drawn in Figure 8. Arguing as in the inner Abelian integral we can obtain that the outer Abelian integral has two or three simple zeros depending on the values of $(\rho, \eta)$.

All the above results, together with the obtained in Proposition 3.5.(a), can be summarized in the bifurcation diagram given in the statement. See Figure 6. More concretely, the dark gray region corresponds to the maximal number of 12 simple zeros in configuration $(9,3)$. The region with 11 simple zeros correspond with the medium gray and the light gray in configurations $(8,3)$ and $(9,2)$, respectively. The cases with 10 simple zeros in configuration $(8,2)$ correspond to the white region. 


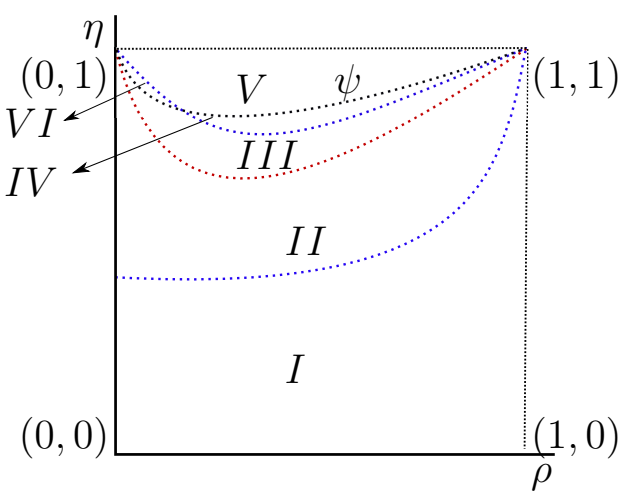

Figure 7 . The zero level curves of $N_{e, 0}, N_{e, \eta}$ and $D_{e, 1}$ in black, red, and blue dotted lines, respectively

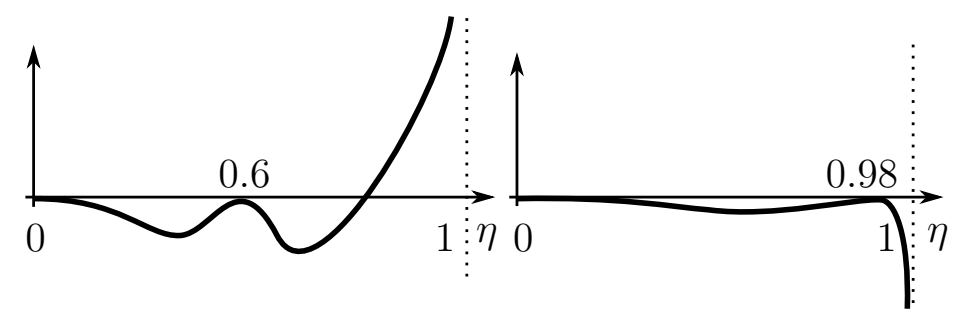

Figure 8. The graphs of $I_{e}(r, \hat{\alpha})$ for $(\rho, \eta)=(0.1,0.6)$ and $(\rho, \eta)=$ $(0.4,0.98)$, respectively

From the above proof we have seen that the bifurcation curves are the numerators and denominators of the series expansions in $\rho$ and $\eta$. In particular they are functions of the form (17). To simplify the reading, we have unified how the bifurcation curves that will appear in the following results are written,

$$
\begin{aligned}
N_{j, \xi}(\rho, \eta)= & p_{0}^{j, \xi}+p_{1}^{j, \xi} L(\eta)+p_{2}^{j, \xi} L(\rho)+p_{3}^{j, \xi}(L(\eta))^{2}+p_{4}^{j, \xi} L(\rho) L(\eta), \\
& +p_{5}^{j, \xi} L(\rho)(L(\eta))^{2}+p_{6}^{j, \xi}(L(\eta))^{3}+p_{7}^{j, \xi} L(\rho)(L(\eta))^{3}, \\
D_{j, \xi}(\rho, \eta)= & q_{0}^{j, \xi}+q_{1}^{j, \xi} L(\eta)+q_{2}^{j, \xi} L(\rho)+q_{3}^{j, \xi}(L(\eta))^{2}+q_{4}^{j, \xi} L(\rho) L(\eta), \\
& +q_{5}^{j, \xi} L(\rho)(L(\eta))^{2}+q_{6}^{j, \xi}(L(\eta))^{3}+q_{7}^{j, \xi} L(\rho)(L(\eta))^{3},
\end{aligned}
$$

where $p_{k}^{j, \xi}$ and $q_{k}^{j, \xi}$ are polynomials in $\rho, \eta$ for $j \in\{i, e\}, \xi \in\{0, \rho, 1\}$ and $k=0, \ldots, 7$. As in the last proof all $p_{k}^{j, \xi}$ and $q_{k}^{j, \xi}$ are polynomials of high degrees with rational coefficients. In the next propositions we only get explicitly the sequence of degrees of them because of their sizes. We will say that a polynomial has degree $\hat{0}$ when it vanishes identically.

Proposition 4.3. Let $\rho, \eta$ be zeros of multiplicity 7 and 3 of $I_{i}$ and $\widetilde{I}_{e}$, respectively. There exist three zero level curves $\Theta=\left\{N_{i, \rho}(\rho, \eta)=0\right\}, \Lambda=\left\{N_{i, 0}(\rho, \eta)=0\right\}$, and $\Psi=$ $\left\{N_{e, 0}(\rho, \eta)=0\right\}$ such that the square $(0,1)^{2}$ is divided in four regions, see Figure 9. Moreover, the functions $N_{i, \rho}, N_{i, 0}$ and $N_{e, 0}$ write as (18) and the sequences of degrees of $p_{k}^{i, \rho}, p_{k}^{i, 0}$, and $p_{k}^{e, 0}$ are $\{29,30,26, \hat{0}, 27, \hat{0}, \hat{0}, \hat{0}\},\{29,30,38, \hat{0}, 29, \hat{0}, \hat{0}, \hat{0}\}$, and $\{36,37,37,34,38,25, \hat{0}, \hat{0}\}$ for $k=0, \ldots, 7$, respectively. Then, the configurations of simple zeros of $I_{i}$ and $\widetilde{I}_{e}$, in a neighborhood of $(\rho, \eta)$, are $(9,3),(7,4),(8,3)$, and $(7,3)$, respectively. Moreover, each of them is realizable only in one of the four regions.

Proof. The proof follows similarly as the proof of Proposition 4.2. The main changes are the multiplicities at $\rho$ and $\eta$ that are 7 and 3 and the local behaviors of $I_{i}$ and $\widetilde{I}_{e}$ are also 


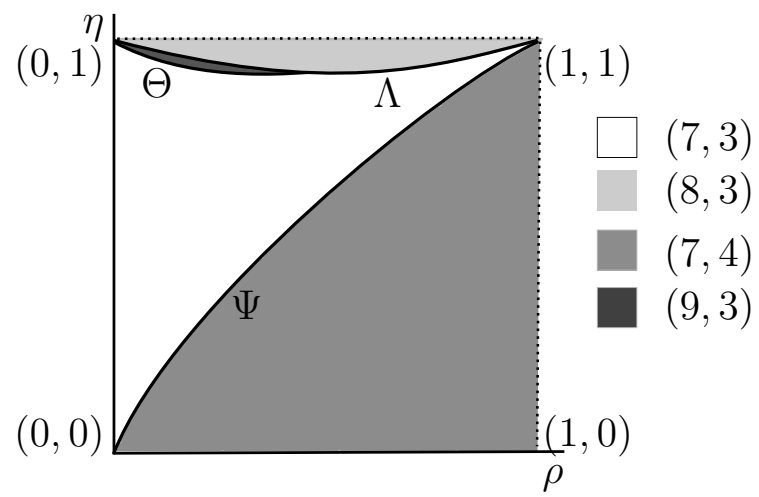

Figure 9. Bifurcation diagram of configuration of simultaneous zeros for the maximum multiplicity case $(7,3)$

necessary to be done. This study, similar to the one performed for the outer function of the previous proposition, gives relations between the numerators and the denominators of the series expansions near $r=0, r=\rho, r=\eta$, and $r=1$. Therefore, we have only three zero level curves corresponding to $I_{i}$ and another three to $\widetilde{I}_{e}$. They are drawn in Figure 10 as dashed and dotted lines, respectively. All these curves are defined as zero level curves of functions of type (18). The remaining sequences of the degrees of the polynomials in (18), among the given in the statement, are $\{31,28,32, \hat{0}, 29, \hat{0}, \hat{0}, \hat{0}\},\{41,40,42,35,41,36, \hat{0}, \hat{0}\}$, and $\{36,37,37,34,38,35, \hat{0}, \hat{0}\}$ for $D_{i, 1}(\rho, \eta), N_{e, \eta}(\rho, \eta)$ and $D_{e, 1}(\rho, \eta)$, respectively. The proof finishes studying the plot of the functions $I_{i}$ and $\widetilde{I}_{e}$ in each region depicted in Figure 10.

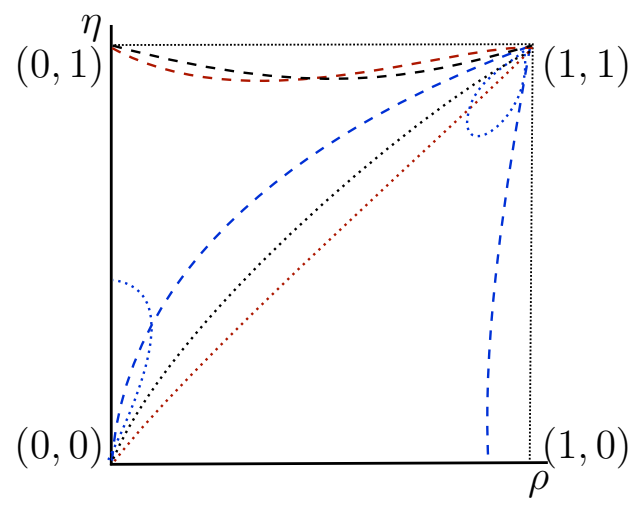

Figure 10. The zero level curves of $N_{i, 0}, N_{i, \rho}, D_{i, 1}$ (dashed line) and $N_{e, 0}$, $N_{e, \eta}, D_{e, 1}$ (dotted line) in black, red, and blue, respectively

Proposition 4.4. Let $\rho, \eta$ be zeros of multiplicity 6 and 4 of $I_{i}$ and $\widetilde{I}_{e}$, respectively. There exist three level curves $\Lambda=\left\{N_{i, 0}(\rho, \eta)=0\right\}, \Psi=\left\{N_{e, 0}(\rho, \eta)=0\right\}$, and $\Phi=$ $\left\{N_{e, \eta}(\rho, \eta)=0\right\}$, such that the square $(0,1)^{2}$ is divided in four regions, see Figure 11. Moreover, the functions $N_{i, 0}, N_{e, 0}$, and $N_{e, \eta}$, write as (18) and the list of degrees of the polynomials $p_{k}^{i, 0}, p_{k}^{e, 0}$, and $p_{k}^{e, \eta}$ are $\{34,35,35,32,32,31, \hat{0}, \hat{0}\},\{38,39,39,38,38,33,33,32\}$, and $\{42,43,43,40,40,33, \hat{0}, \hat{0}\}$, for $k=0, \ldots, 7$, respectively. Then, the configurations of simple zeros of $I_{i}$ and $\widetilde{I}_{e}$, in a neighborhood of $(\rho, \eta)$, are $(6,6),(7,4),(6,5)$, and $(6,4)$, respectively. Each of them is realizable only in one of the four regions.

Proof. The proof follows as the proof of Proposition 4.3 changing only the multiplicities, that are 6 and 4 , and the zero level curves. In particular, the sequences of degrees, among the ones detailed in the statement, are $\{38,37,39,32,38,33, \hat{0}, \hat{0}\},\{38,37,37,32,32,31, \hat{0}, \hat{0}\}$, 


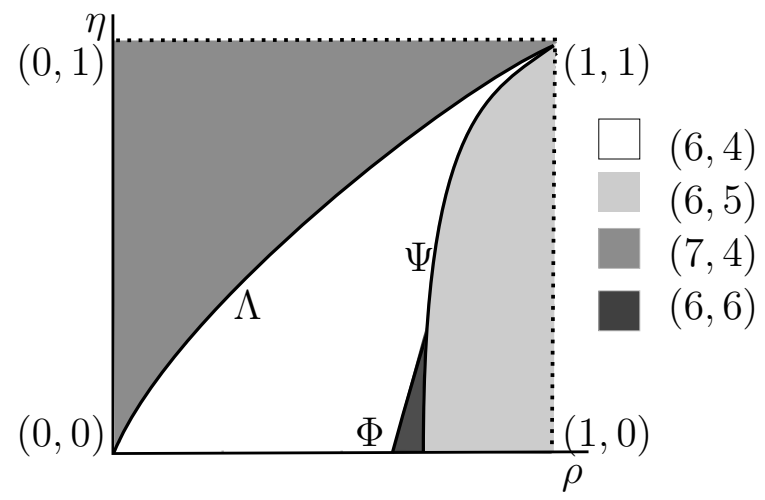

Figure 11. Bifurcation diagram of configuration of simultaneous zeros for the maximum multiplicity case $(6,4)$

and $\{38,39,37,38,38,33,33,32\}$, for $N_{i, \rho}(\rho, \eta), D_{i, 1}(\rho, \eta)$, and $D_{e, 1}(\rho, \eta)$, respectively. The zero level curves for this case are drawn in Figure 12.

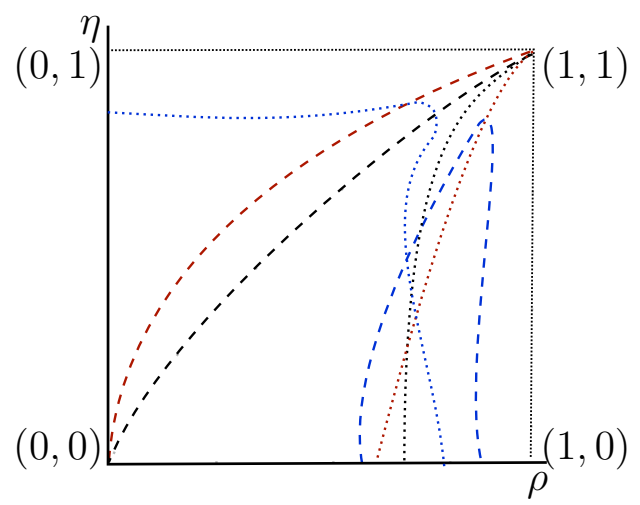

Figure 12. The zero level curves of $N_{i, 0}, N_{i, \rho}, D_{i, 1}$ (dashed line) and $N_{e, 0}$, $N_{e, \eta}, D_{e, 1}$ (dotted line) in black, red, and blue, respectively

Proposition 4.5. Let $\rho, \eta$ be zeros of multiplicity 5 of $I_{i}$ and $\widetilde{I}_{e}$, respectively. There exist two level curves $\Lambda=\left\{N_{i, 0}(\rho, \eta)=0\right\}$ and $\Psi=\left\{N_{e, 0}(\rho, \eta)=0\right\}$, such that the square $(0,1)^{2}$ is divided in four regions, see Figure 13. Moreover, the functions $N_{0, i}$ and $N_{0, e}$ write as (18) and the sequences of degrees of $p_{k}^{i, 0}$ and $p_{k}^{e, 0}$ are $\{30,31,31,30,30,25, \hat{0}, \hat{0}\}$ and $\{33,34,34,35,35,32,32,25\}$ for $k=0, \ldots, 7$, respectively. Then, the configurations of simple zeros of $I_{i}$ and $\widetilde{I}_{e}$, in a neighborhood of $(\rho, \eta)$, are $(6,6),(6,5),(6,5)$, and $(5,5)$, respectively. Each of them is realizable only in one of the four regions.

Proof. The proof follows as the proof of Proposition 4.3. Here the multiplicities are 5 in both inner and outer regions. Moreover, the sequences of degrees, among the ones detailed in the statement, are $\{38,39,39,36,36,29, \hat{0}, \hat{0}\},\{34,35,35,32,32,25, \hat{0}, \hat{0}\},\{36,37,37,36,36,31, \hat{0}, \hat{0}\}$, and $\{33,34,34,35,35,32,30,25\}$, for $N_{i, \rho}(\rho, \eta), D_{i, 1}(\rho, \eta), N_{e, \eta}(\rho, \eta)$, and $D_{e, 1}(\rho, \eta)$, respectively. The zero level curves for this case are depicted in Figure 14.

Proposition 4.6. Let $\rho, \eta$ be zeros of multiplicity 4 and 6 of $I_{i}$ and $\widetilde{I}_{e}$, respectively. There exist four level curves $\Theta=\left\{N_{i, \rho}(\rho, \eta)=0\right\}, \Lambda=\left\{N_{i, 0}(\rho, \eta)=0\right\}, \Phi=\left\{N_{e, \eta}(\rho, \eta)=0\right\}$, and $\Psi=\left\{N_{e, 0}(\rho, \eta)=0\right\}$, such that the square $(0,1)^{2}$ is divided in five regions, see Figure 15. Moreover, the functions $N_{i, \rho}, N_{i, 0}, N_{e, \eta}$, and $N_{e, 0}$, write as (18) and the sequences 


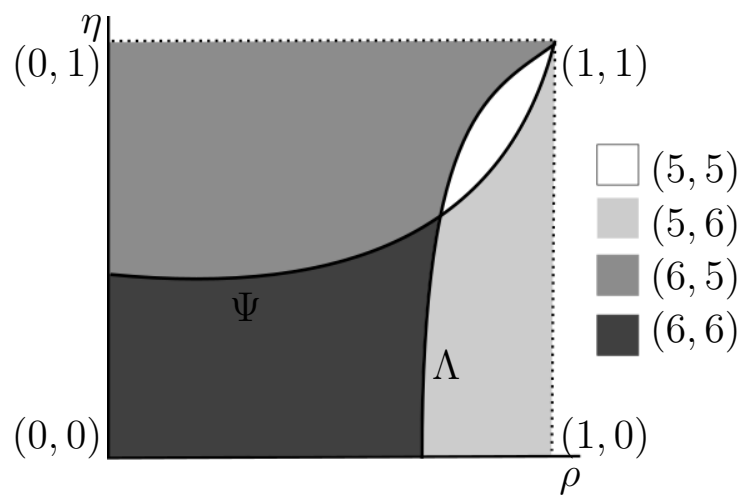

FiguRE 13. Bifurcation diagram of configuration of simultaneous zeros for the maximum multiplicity case $(5,5)$

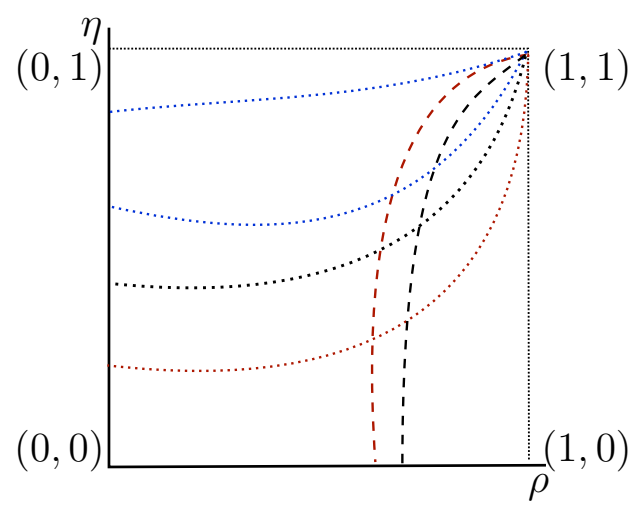

Figure 14. The zero level curves of $N_{i, 0}, N_{i, \rho}, D_{i, 1}$ (dashed line) and $N_{e, 0}$, $N_{e, \eta}, D_{e, 1}$ (dotted line) in black, red and blue, respectively

of degrees of $p_{k}^{i, \rho}, p_{k}^{i, 0}, p_{k}^{e, \eta}$, and $p_{k}^{e, 0}$, are $\{32,33,33,32,32,27, \hat{0}, \hat{0}\},\{24,25,23,22,24,21, \hat{0}, \hat{0}\}$, $\{31,32,30,29,31,28, \hat{0}, \hat{0}\}$, and $\{27,28,28,29,29,28,24,23\}$ are $k=0, \ldots, 7$, respectively. Then, the configurations of simple zeros of $I_{i}$ and $\widetilde{I}_{e}$, in a neighborhood of $(\rho, \eta)$, are $(4,8)$, $(6,6),(4,7),(5,6)$, and $(4,6)$, respectively. Each of them is realizable only in one of the five regions.

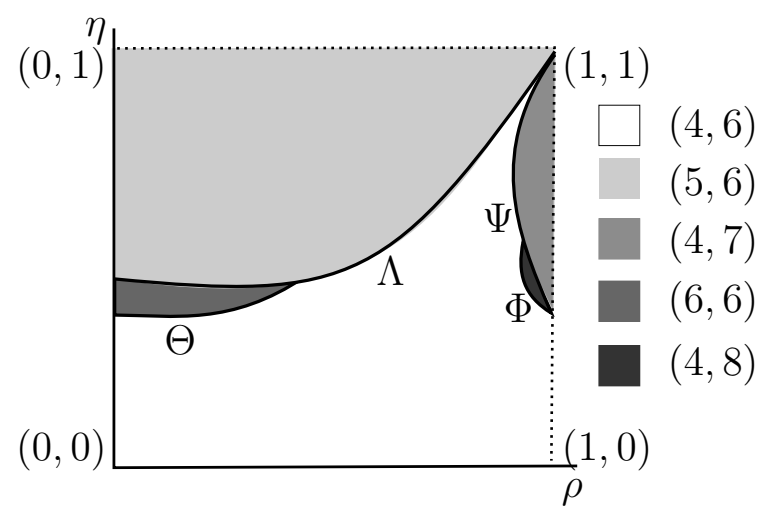

FIGURE 15. Bifurcation diagram of configuration of simultaneous zeros for the maximum multiplicity case $(4,6)$

Proof. The proof follows, changing the multiplicities to 4 and 6, as the proof of Proposition 4.3. The sequences of degrees, among the ones detailed in the statement, are $\{30,29,29,24,28,23, \hat{0}, \hat{0}\}$, and $\{29,30,28,29,29,28,22,23\}$ for $D_{i, 1}(\rho, \eta)$ and $D_{e, 1}(\rho, \eta)$, respectively. The zero level curves for this case are drawn in Figure 16. 


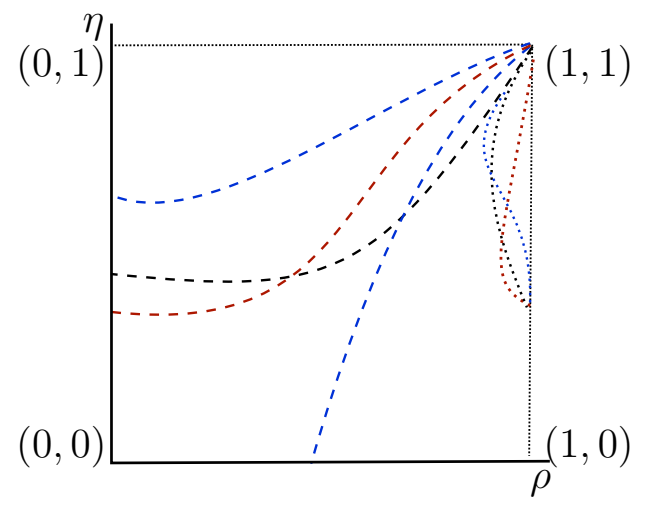

Figure 16. The zero level curves of $N_{i, 0}, N_{i, \rho}, D_{i, 1}$ (dashed line) and $N_{e, 0}$, $N_{e, \eta}, D_{e, 1}$ (dotted line) in black, red, and blue, respectively

Proposition 4.7. Let $\rho, \eta$ be zeros of multiplicity 3 and 7 of $I_{i}$ and $\widetilde{I}_{e}$, respectively. There exists one level curve $\Gamma=\left\{\eta^{*} \approx 0.57704\right\}$, such that the square $(0,1)^{2}$ is divided in two regions, see Figure 17. Then, the configurations of simple zeros of $I_{i}$ and $\widetilde{I}_{e}$, in a neighborhood of $(\rho, \eta)$, are $(4,8)$ and $(4,7)$, respectively. Each of them is realizable only in one of the two regions, see also Figure 17.

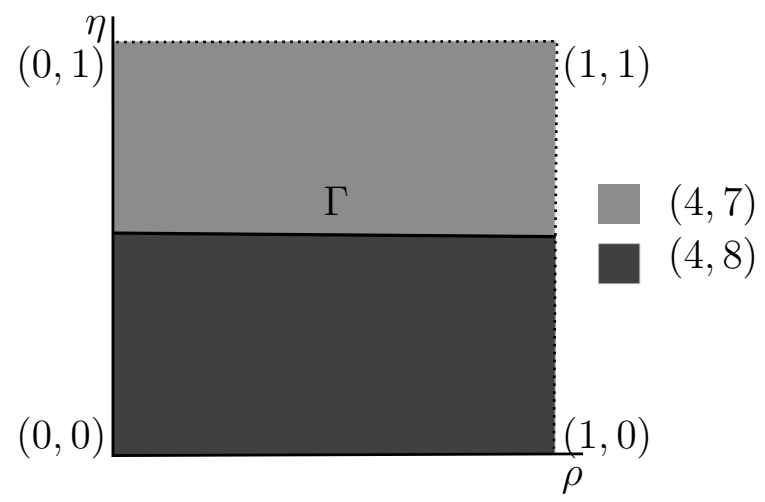

FiguRE 17. Bifurcation diagram of configuration of simultaneous zeros for the maximum multiplicity case $(3,7)$

Proof. The proof follows as the proof of Proposition 4.3 changing only the multiplicities, that in this case are 3 and 7 , and the zero level curves. The sequences of degrees are $\{29,30,28,27,29,26, \hat{0}, \hat{0}\},\{14,15, \hat{0}, 14, \hat{0}, \hat{0}, \hat{0}, \hat{0}\}$, and $\{20,21, \hat{0}, 18, \hat{0}, \hat{0}, \hat{0}, \hat{0}\}$, for $N_{i, \rho}(\rho, \eta)$, $N_{i, 0}(\rho, \eta)$, and $D_{i, 1}(\rho, \eta)$, respectively. The zero level curves are depicted in Figure 18. In fact in the square $(0,1)^{2}$ only appear two curves.

Finally we summarize the configurations given in all the above results in the next corollary.

Corollary 4.8. There exist polynomial perturbation of degree 3 such that (1) exhibits 10 , 11 , and 12 limit cycles in configurations $\{(8,2),(7,3),(6,4),(5,5),(4,6),(3,7)\},\{(9,2)$, $(8,3),(7,4),(6,5),(5,6),(4,7)\}$, and $\{(9,3),(6,6),(4,8)\}$, respectively.

From the technique used in this section it is clear that, as there are 10 essential parameters, there exist perturbations such that system (1) exhibits all the configurations of limit cycles of type $(k, \ell)$ with $k+\ell<10,0 \leq k \leq 8$ and $0 \leq \ell \leq 7$. For that reason we have concentrated our efforts in the configurations exhibiting more limit cycles and how they can appear. 


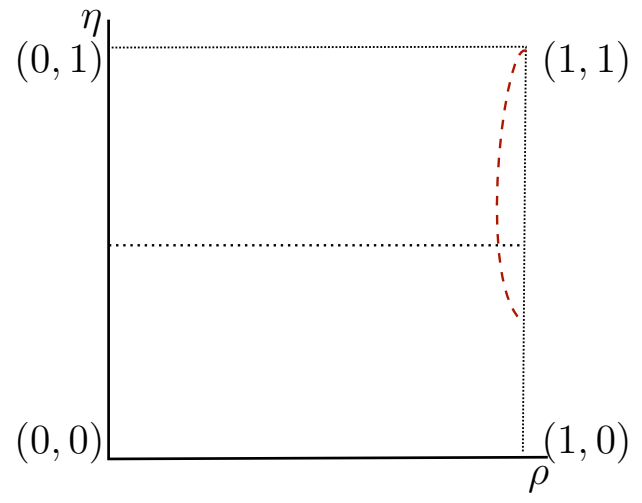

Figure 18. The zero level curves of $N_{i, \rho}$ (dashed line) in red and $N_{e, 0}$ (dotted line) in black, respectively

\section{LOCAL STUdy OF THE ZERO LEVEL CURVES IN THE BIFURCATION DIAGRAMS}

Finally, we explain how the zero levels curves (17) can be studied near the boundary of the domain of definition and the difficulties, that we have found, when they are non analytic. We make the analysis in the neighborhood of the corners of $[0,1]^{2}$ where they can be extended. Before the description of them, we present a general result about how to obtain the branches of a curve with only one logarithm function.

Lemma 5.1. Consider the zero level curve

$$
f(u, v)+g(u, v) \log v=0,
$$

with $f$ and $g$ analytical functions such that $f(0,0)=g(0,0)=0$. Then, it is a solution of the differential equation

$$
\left\{\begin{array}{l}
\dot{u}=h(u, v), \\
\dot{v}=v,
\end{array}\right.
$$

where $h=\frac{v\left(f g_{v}-f_{v} g\right)-g^{2}}{f_{u} g-f g_{u}}$. Furthermore, if $h$ is analytical, $h(0,0)=0$, and $\frac{\partial h}{\partial u}(0,0)=k$, with $k$ a natural bigger than one, then there exists a $\mathcal{C}^{\infty}$ function $U(u, v)$ such that $u(v)=$ $U\left(v^{k} \log v, v\right)$ satisfies (19).

Proof. Firstly, we consider a $t$-parameterization, $(u(t), v(t))$, of the level curve (19) such that $v \neq 0$. As it satisfies $\frac{\mathrm{d}}{\mathrm{d} t}(f(u(t), v(t))+g(u(t), v(t)) \log (v(t)))=0$ for every $t$, we can write

$$
f_{u} \dot{u}+f_{v} \dot{v}+\left(g_{u} \dot{u}+g_{v} \dot{v}\right) \log v+g \frac{1}{v} \dot{v}=f_{u} \dot{u}+f_{v} \dot{v}-\left(g_{u} \dot{u}+g_{v} \dot{v}\right) \frac{f}{g}+g \frac{1}{v} \dot{v}=0,
$$

or equivalently,

$$
v\left(f_{u} g-f g_{u}\right) \dot{u}+\left(v\left(f_{v} g-f g_{v}\right)+g^{2}\right) \dot{v}=0 .
$$

So, we have written the level curve as a solution of system (20).

Secondly, under the conditions of the statement, the origin is an equilibrium point of (20) such that the matrix of its linear part has eigenvalues $k$ and 1 . The study of the normal form of (20) can be done using the resonance theory. In fact, only the first component, $h(u, v)$, has resonances. As the equation $(k, 1)(n, m)-k=0$ has solution only when $(n, m)=(0, k)$, then there exists a diffeomorphism $(u, v)=(U(x, y), y)$ that transforms system (20) to its normal form

$$
\left\{\begin{array}{l}
\dot{x}=k x-y^{k}, \\
\dot{y}=y .
\end{array}\right.
$$


The proof finishes using $(x(t), y(t))=\left(\left(x_{0}-y_{0}^{k} t\right) e^{k t}, y_{0} e^{t}\right)$ as the solution of the above differential equation and changing $t=\log \left(y / y_{0}\right)=\log \left(v / y_{0}\right)$.

Proposition 5.2. Let $N_{e, 0}, N_{e, \eta}$, and $D_{e, 1}$ be the zero level curves defined in (17). Then,

(a) there are only two branches passing through $(0,0)$ and they are $\rho=\eta=0$,

(b) there is only one branch passing through $(1,0)$ and it is $\eta=0$,

(c) for each $N_{e, 0}, N_{e, \eta}$, and $D_{e, 1}$, there is only one branch passing through $(0,1)$. Their series expansions are

$$
\begin{aligned}
0 & =\rho+\frac{945}{32} L(\eta)\left(\frac{1-\eta}{1+\eta}\right)^{2}-\frac{315}{64}\left(\frac{1-\eta}{1+\eta}\right)+\frac{315}{64}\left(\frac{1-\eta}{1+\eta}\right)^{2}+\frac{2835}{16} L(\eta)\left(\frac{1-\eta}{1+\eta}\right)^{3}+\cdots \\
0 & =\rho+\frac{945}{32} L(\eta)\left(\frac{1-\eta}{1+\eta}\right)^{3}-\frac{315}{128}\left(\frac{1-\eta}{1+\eta}\right)+\frac{315}{64}\left(\frac{1-\eta}{1+\eta}\right)^{2}+\frac{945}{4} L(\eta)\left(\frac{1-\eta}{1+\eta}\right)^{4}+\cdots \\
0 & =\rho+\frac{315}{8} L(\eta)\left(\frac{1-\eta}{1+\eta}\right)^{2}-\frac{315}{64}\left(\frac{1-\eta}{1+\eta}\right)+\frac{105}{2}\left(\frac{1-\eta}{1+\eta}\right)^{2}+\frac{945}{16} L(\eta)\left(\frac{1-\eta}{1+\eta}\right)^{3}+\cdots
\end{aligned}
$$

respectively.

(d) for each $N_{e, 0}, N_{e, \eta}$ there is only one branch passing through $(1,1)$ and two for $D_{e, 1}$. Their series expansion are

$$
\begin{aligned}
0 & =\frac{1-\eta}{1+\eta}-\frac{1}{2} \frac{\sqrt{10}}{\sqrt{-L(\rho)}}\left(\frac{1-\rho}{1+\rho}\right)^{2}+\cdots, \\
0 & =\frac{1-\eta}{1+\eta}-\frac{1}{2} \frac{\sqrt[3]{20}}{\sqrt[3]{-L(\rho)}}\left(\frac{1-\rho}{1+\rho}\right)^{4 / 3}+\cdots, \\
0 & =\frac{1-\eta}{1+\eta}-\sqrt{3}\left(\frac{1-\rho}{1+\rho}\right)^{2}+\cdots, \\
0 & =\frac{1-\eta}{1+\eta}+\frac{5}{12} \frac{1}{L(\rho)}+\cdots
\end{aligned}
$$

respectively.

Proof. (a) All curves in (17) are analytic in $(\rho, \eta)=(0,0)$ and, in order to unify notation along the proof, we write $(\rho, \eta)=(u, v)$. Now, at the origin, they write as $f(u, v)=$ $u^{i} v^{j} \widetilde{f}(u, v)$ with $\widetilde{f}(0,0) \neq 0$. More concretely, we have

$$
\begin{aligned}
& \widetilde{N}_{e, 0}=\frac{4096}{21} u^{8} v^{7}\left(63+224 u^{2}-90 v^{2}+\cdots\right), \\
& \widetilde{N}_{e, \eta}=-\frac{4096}{21} u^{8} v^{9}\left(63+224 u^{2}-81 v^{2}+\cdots\right), \\
& \widetilde{D}_{e, 1}=-\frac{4096}{21} u^{8} v^{5}\left(63+224 u^{2}-297 v^{2}+\cdots\right) .
\end{aligned}
$$

So, moving to the original coordinates, all curves has only the branches $\rho=\eta=0$ at the origin.

(b) The study near $(\rho, \eta)=(1,0)$ will be done near the origin, as in the previous case, but using the change $(\rho, \eta)=\left(\frac{1-u}{1+u}, v\right)$. Although the curves are analytic only with respect to $\eta$, or $v$, the series, using the logarithmic function, can be extended to the origin. They write as $f(u, v)=v^{j} \widetilde{f}(u, v)$ with $\widetilde{f}(u, v)$ different from zero near the origin. More 
concretely, we have

$$
\begin{aligned}
& \widetilde{N}_{e, 0}=4096 v^{7}\left(-12 \log u-25+24 v^{2} \log u+\cdots\right), \\
& \widetilde{N}_{e, \eta}=-4096 v^{9}\left(-12 \log u-25+36 v^{2} \log u+\cdots\right), \\
& \widetilde{D}_{e, 1}=4096 v^{5}\left(-12 \log u-25+36 v^{2} \log u+\cdots\right) .
\end{aligned}
$$

The proof of this statement finishes because, in the above curves only $v=0$, or $\eta=0$, passes through the point $(1,0)$.

(c) As in the above cases, we study the neighborhood of the point $(\rho, \eta)=(0,1)$ with an adequate change of variables, $(\rho, \eta)=\left(u, \frac{1-v}{1+v}\right)$, that moves it to the origin. But in this case we use also Lemma 5.1 for each function $N_{e, 0}, N_{e, \eta}$ and $D_{e, 1}$, writing them in the form $f(u, v)=u^{i} \widetilde{f}(u, v)$, where $\widetilde{f}(u, v)=\widetilde{f}_{0}(u, v)+\widetilde{f}_{1}(u, v) \log v$ and $\widetilde{f}_{0}(0,0)=\widetilde{f}_{1}(0,0)=0$.

Firstly, we detail the computations for $N_{e, 0}$, in (17). For this function, we have

$$
\begin{aligned}
& \widetilde{f}_{0}(u, v)=704 u-3465 v+72765 v^{2}-14080 u v+\cdots, \\
& \widetilde{f}_{1}(u, v)=9 v^{2}-126 v^{3}+936 v^{4}+4 u^{2} v^{2}+\cdots .
\end{aligned}
$$

Hence, applying Lemma 5.1, we get (20) with $k=2$ and

$$
h(u, v)=2 u-\frac{315}{64} v-\frac{5985}{128} v^{2}+\frac{3}{2} u v+\cdots .
$$

Consequently, there exists a diffeomorphism $(u, v)=(U(x, y), y)$ such that the normal form associated to (20) is

$$
(\dot{x}, \dot{y})=\left(2 x-\frac{5985}{128} y^{2}, y\right)
$$

which has the solution

$$
(x(t), y(t))=\left(\left(-\frac{5985}{128} y_{0}^{2} t+x_{0}\right) e^{2 t}, y_{0} e^{t}\right) .
$$

Notice that, the above normal form is equivalent, after a rescaling in $y$, to (21). Then, we get $u=U\left(\left(-\frac{5985}{128} y_{0}^{2} t+x_{0}\right) e^{2 t}, y_{0} e^{t}\right)$ and $u=U\left(v^{2} \log v, v\right)$. From this normal form we can write also the series expansion near the origin, that is

$$
u=\frac{315}{64} v-\frac{945}{32} v^{2} \log v-\frac{315}{64} v^{2}-\frac{2835}{16} v^{3} \log v+\cdots .
$$

The expression in the statement follows recovering the original coordinates, $(\rho, \eta)$.

Secondly, for $N_{e, \eta}$, the resonance corresponding to the normal form for equation (20) is $k=3$ and the series expansion writes as

$$
u=\frac{315}{128} v-\frac{315}{64} v^{2}-\frac{945}{32} v^{3} \log v-\frac{945}{4} v^{4} \log v+\cdots .
$$

Finally, as in the above cases, for $D_{e, 1}$ we obtain $k=2$ and

$$
u=-\frac{315}{64} v+\frac{315}{8} v^{2} \log v+\frac{105}{2} v^{2}+\frac{945}{16} v^{3} \log v+\cdots .
$$

(d) The last case is the study of the behavior of $(\rho, \eta)=(1,1)$. The translation to the origin now is done by the change of variables $(\rho, \eta)=\left(\frac{1-u}{1+u}, \frac{1-v}{1+v}\right)$. But for this last case is more difficult to find a general local development of all the curves. So, we will find only the first terms using a generalized Newton's diagram, using the powers in the variables $u, v, \log u$, and $\log v$, see [15]. In fact we depict the terms of the form $u^{n_{1}} v^{m_{1}}, u^{n_{2}} v^{m_{2}} \log u$, $u^{n_{3}} v^{m_{3}} \log v$ and $u^{n_{4}} v^{m_{4}} \log u \log v$ for our three curves to see which of them are enough to describe the series expansion near the origin. The corresponding generalized Newton's diagrams for the functions $\widetilde{N}_{e, 0}, \widetilde{N}_{e, \eta}$, and $\widetilde{D}_{e, 1}$ are drawn in Figure 19. Hence, from them, 

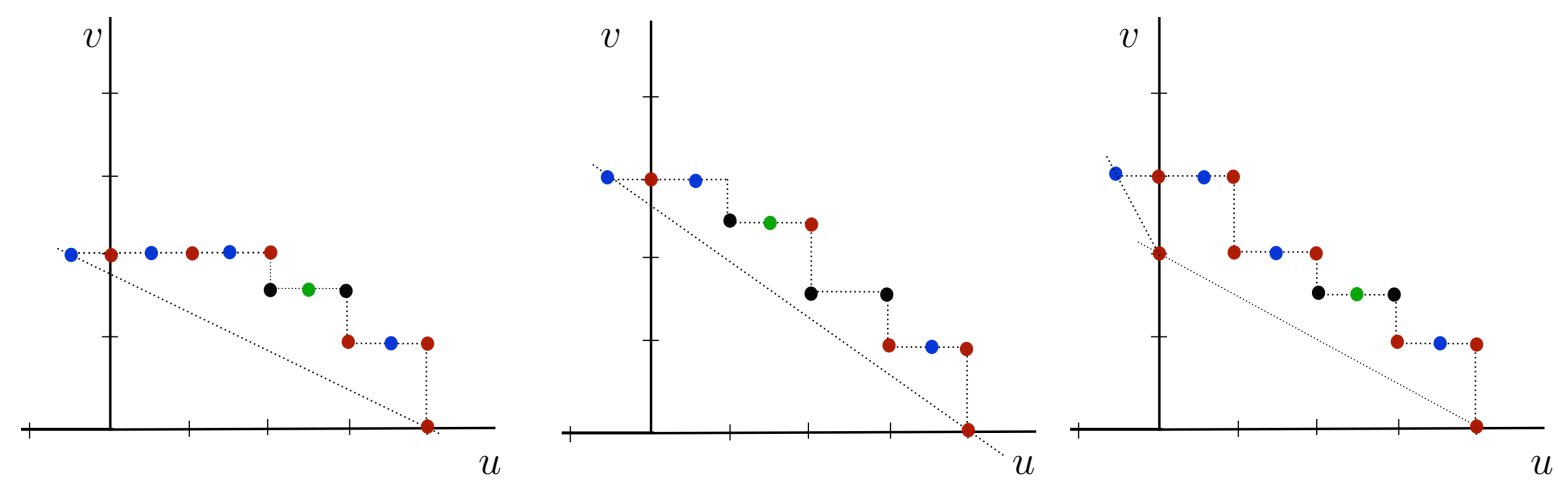

FiguRE 19. The red, blue, black and green are points represent the monomials of $N_{e, 0}$ (left), $N_{e, \eta}$ (middle), and $D_{e, 1}$ (right) associated to $\left(u^{n_{1}} v^{m_{1}}\right)$, $\left(u^{n_{2}} v^{m_{2}} \log u\right),\left(u^{n_{3}} v^{m_{3}} \log u\right)$ and $\left(u^{n_{4}} v^{m_{4}} \log u \log v\right)$, respectively.

the first terms for studying the different branches of the level zero curve are

$$
\begin{aligned}
& \widetilde{N}_{e, 0}=-393216\left(2 v^{2} \log u+5 u^{4}\right)+\cdots, \\
& \widetilde{N}_{e, \eta}=1572864\left(2 v^{3} \log u+5 u^{4}\right)+\cdots, \\
& \widetilde{D}_{e, 1}=-262144\left(12 v^{3} \log u+5 v^{2}-15 u^{4}\right)+\cdots .
\end{aligned}
$$

Then, the branches of $\widetilde{N}_{e, 0}=0$ follow studying a series expansion of the form $v=$ $A_{0} u^{2} / \sqrt{-\log u}+\cdots$. Straightforward computations give us two possible values for $A_{0}$, $\pm \sqrt{10} / 2$, but, as we are interested only in the branches in the first quadrant $(u, v>0)$, we get $A_{0}=\sqrt{10} / 2$. The branches of $\widetilde{N}_{e, \eta}=0$ follow in a similar way, but with series expansion of the form $v=A_{\eta} u^{4 / 3} /(-\log u)^{1 / 3}+\cdots$. In this case we have only one possible real value for $A_{\eta}=20^{1 / 3} / 2$. For $\widetilde{D}_{e, 1}=0$, we have to study two type of different branches $v=A_{1,1} u^{2}+\cdots$ and $v=A_{1,2} / \log u+\cdots$. The possible values for $A_{1,1}$ and $A_{1,2}$ are $\pm \sqrt{3}$ and $5 / 12$, respectively. But, restricting to the first quadrant, we have only one for each, $A_{1,1}=\sqrt{3}$ and $A_{1,2}=5 / 12$. The expressions that appear in the statement follow recovering the original coordinates.

Finally, we show the existence of an intersection point for the level zero curves $N_{e, 0}$ and $D_{e, 1}$, defined in (17), as we have depicted in Figure 7. This fact follows just comparing their series expansions near the points $(0,1)$ and $(1,1)$. Numerically it is not so easy to find this intersection point. But, transforming the system to the equivalent one

$$
\begin{gathered}
\frac{q_{0}^{1}(\rho, \eta)}{q_{3}^{1}(\rho, \eta)}-\frac{p_{0}^{0}(\rho, \eta)}{p_{3}^{0}(\rho, \eta)}+\left(\frac{q_{1}^{1}(\rho, \eta)}{q_{3}^{1}(\rho, \eta)}-\frac{p_{1}^{0}(\rho, \eta)}{p_{3}^{0}(\rho, \eta)}\right) L(\rho)+\left(\frac{q_{2}^{1}(\rho, \eta)}{q_{3}^{1}(\rho, \eta)}-\frac{p_{2}^{0}(\rho, \eta)}{p_{3}^{0}(\rho, \eta)}\right) L(\eta)=0, \\
\frac{q_{0}^{1}(\rho, \eta)}{q_{1}^{1}(\rho, \eta)}-\frac{p_{0}^{0}(\rho, \eta)}{p_{1}^{0}(\rho, \eta)}+\left(\frac{q_{2}^{1}(\rho, \eta)}{q_{1}^{1}(\rho, \eta)}-\frac{p_{2}^{0}(\rho, \eta)}{p_{1}^{0}(\rho, \eta)}\right) L(\eta)+\left(\frac{q_{3}^{1}(\rho, \eta)}{q_{1}^{1}(\rho, \eta)}-\frac{p_{3}^{0}(\rho, \eta)}{p_{1}^{0}(\rho, \eta)}\right) L(\rho) L(\eta)=0,
\end{gathered}
$$

we numerically can find the intersection point $(\rho, \eta) \approx(0.0325224964,0.9889101365)$.

\section{ACKNOWLEDGEMENTS}

The first author is supported by CAPES BEX 13473/13-1. All authors are supported by the MINECO grants MTM2013-40998-P and MTM2016-77278-P (FEDER) and the AGAUR grant 2014 SGR568. 


\section{REFERENCES}

[1] A. Buică and J. Llibre. Averaging methods for finding periodic orbits via Brouwer degree. Bull. Sci. Math., 128(1):7-22, 2004.

[2] C. Buzzi, C. Pessoa, and J. Torregrosa. Piecewise linear perturbations of a linear center. Discrete Contin. Dyn. Syst., 33(9):3915-3936, 2013.

[3] C. Christopher. Estimating limit cycle bifurcations from centers. In Differential equations with symbolic computation, Trends Math., pages 23-35. Birkhäuser, Basel, 2005.

[4] C. Christopher and C. Li. Limit cycles of differential equations. Advanced Courses in Mathematics. CRM Barcelona. Birkhäuser Verlag, Basel, 2007.

[5] C. J. Christopher and N. G. Lloyd. Polynomial systems: a lower bound for the Hilbert numbers. Proc. Roy. Soc. London Ser. A, 450(1938):219-224, 1995.

[6] B. Coll, C. Li, and R. Prohens. Quadratic perturbations of a class of quadratic reversible systems with two centers. Discrete Contin. Dyn. Syst., 24(3):699-729, 2009.

[7] F. Dumortier and C. Li. Perturbation from an elliptic Hamiltonian of degree four. IV. Figure eightloop. J. Differential Equations, 188(2):512-554, 2003.

[8] P. Fatou. Sur le mouvement d'un système soumis à des forces à courte période. Bull. Soc. Math. France, 56:98-139, 1928.

[9] A. Garijo, A. Gasull, and X. Jarque. Simultaneous bifurcation of limit cycles from two nests of periodic orbits. J. Math. Anal. Appl., 341(2):813-824, 2008.

[10] A. Gasull, J. Tomás Lázaro, and J. Torregrosa. Upper bounds for the number of zeroes for some Abelian integrals. Nonlinear Anal., 75(13):5169-5179, 2012.

[11] A. Gasull and J. Torregrosa. Center-focus problem for discontinuous planar differential equations. Internat. J. Bifur. Chaos Appl. Sci. Engrg., 13(7):1755-1765, 2003. Dynamical systems and functional equations (Murcia, 2000).

[12] S. Karlin and W. J. Studden. Tchebycheff systems: With applications in analysis and statistics. Pure and Applied Mathematics, Vol. XV. Interscience Publishers John Wiley \& Sons, New York-LondonSydney, 1966.

[13] A. Khovanskii. Cycles of dynamical systems on the plane and Rolle's theorem. Siberian Mathematical Journal, 25(3):502-506, 1984.

[14] J. Li. Hilbert's 16th problem and bifurcations of planar polynomial vector fields. Internat. J. Bifur. Chaos Appl. Sci. Engrg., 13(1):47-106, 2003.

[15] J.-M. Lion and J.-P. Rolin. Théorème de préparation pour les fonctions logarithmico-exponentielles. Ann. Inst. Fourier (Grenoble), 47(3):859-884, 1997.

[16] J. Llibre, A. C. Mereu, and D. D. Novaes. Averaging theory for discontinuous piecewise differential systems. Journal of Differential Equations, 258(11):4007 - 4032, 2015.

[17] J. Llibre, D. D. Novaes, and M. A. Teixeira. On the birth of limit cycles for non-smooth dynamical systems. Bulletin des Sciences Mathématiques, 139(3):229 - 244, 2015.

[18] J. Llibre, J. S. Pérez del Río, and J. A. Rodríguez. Averaging analysis of a perturbated quadratic center. Nonlinear Anal., 46(1, Ser. A: Theory Methods):45-51, 2001.

[19] J. Llibre and E. Ponce. Piecewise linear feedback systems with arbitrary number of limit cycles. Internat. J. Bifur. Chaos Appl. Sci. Engrg., 13(4):895-904, 2003.

[20] H. Ma and M. Han. Limit cycles of a $Z_{3}$-equivariant near-Hamiltonian system. Nonlinear Anal., 71(9):3853-3871, 2009.

[21] H. Ma, M. Han, and C. Lhotka. Limit cycles of some $Z_{3}$-equivariant near-Hamiltonian systems of degrees 3 and 4. Ann. Differential Equations, 25(2):170-178, 2009.

[22] J. C. Medrado and J. Torregrosa. Uniqueness of limit cycles for sewing piecewise linear systems. J. Math. Anal. Appl., 431:529-544, 2015.

[23] D. D. Novaes and J. Torregrosa. On the extended Chebyshev systems with positive accuracy. $J$. Math. Anal. Appl., 448:171-186, 2017.

[24] S. Pérez-González and J. Torregrosa. Simultaneous bifurcation of limit cycles from a linear center with extra singular points. Bull. Sci. Math., 138(1):124-138, 2014.

[25] Y. Xiong. The number of limit cycles in perturbations of polynomial systems with multiple circles of critical points. J. Math. Anal. Appl., 440(1):220-239, 2016. 
Departament de Matemàtiques, Universitat Autònoma de Barcelona, 08193 Bellaterra, Barcelona, Catalonia, Spain

E-mail address: leonardo@mat.uab.cat

Departament de Matemàtiques, Universitat Autònoma de Barcelona, 08193 Bellaterra, Barcelona, Catalonia, Spain

E-mail address: torre@mat.uab.cat 